\title{
The Coordination of Arm Movements: An Experimentally Confirmed Mathematical Model ${ }^{1}$
}

\author{
TAMAR FLASH ${ }^{*, 2}$ AND NEVILLE HOGAN $\ddagger^{3}$ \\ * Department of Psychology and $\ddagger$ Department of Mechanical Engineering, Massachusetts Institute of Technology, Cambridge, \\ Massachusetts 02139
}

\begin{abstract}
This paper presents studies of the coordination of voluntary human arm movements. A mathematical model is formulated which is shown to predict both the qualitative features and the quantitative details observed experimentally in planar, multijoint arm movements.

Coordination is modeled mathematically by defining an objective function, a measure of performance for any possible movement. The unique trajectory which yields the best performance is determined using dynamic optimization theory. In the work presented here, the objective function is the square of the magnitude of jerk (rate of change of acceleration) of the hand integrated over the entire movement. This is equivalent to assuming that a major goal of motor coordination is the production of the smoothest possible movement of the hand.

Experimental observations of human subjects performing voluntary unconstrained movements in a horizontal plane are presented. They confirm the following predictions of the mathematical model: unconstrained point-to-point motions are approximately straight with bell-shaped tangential velocity profiles; curved motions (through an intermediate point or around an obstacle) have portions of low curvature joined by portions of high curvature; at points of high curvature, the tangential velocity is reduced; the durations of the lowcurvature portions are approximately equal.

The theoretical analysis is based solely on the kinematics of movement independent of the dynamics of the musculoskeletal system and is successful only when formulated in terms of the motion of the hand in extracorporal space. The
\end{abstract}

Received July 13, 1984, Revised January 7, 1985;

Accepted January 14, 1985

\footnotetext{
${ }^{1}$ This paper describes research performed in the Department of Psychology and the Artificial Intelligence Laboratory at the Massachusetts Institute of Technology. The research was supported in part by National Institute of Neurological Disease and Stroke Research Grant NS09343, National Institute of Arthritis, Metabolism, and Digestive Diseases Grant AM26710, National Eye Institute Grant EY02621, and the Advanced Research Projects Agency of the Department of Defense under Office of Naval Research Contract N00014-80-C-0505. T. F. was supported by the Whitaker Health Sciences Fund and by the Bantrell Fellowship in Neurosciences. We wish to acknowledge the contributions of Drs. William Abend, Emilio Bizzi, Pierro Morasso, and John Hollerbach who assisted this project in several ways, both through their insightful comments and by making their experimental data available to us.

${ }^{2}$ Present address: Department of Applied Mathematics, The Weizman Institute of Science, Rehovot 76100 , Israel.

${ }^{3}$ To whom correspondence should be addressed.
}

implications with respect to movement organization are discussed.

How is movement control organized? Which variable(s) are controlled? These questions have become a growing concern of motor neurophysiologists (Granit, 1981; Stein, 1982). Although investigations have traditionally focused on single muscle contractions or single-joint movements, these systems cannot reveal the problems confronted by the central nervous system in the control of normal multijoint movements. Even a two-joint motion is vastly more complicated than a single-joint motion; in moving from one point to another, on what basis does the central nervous system select one specific trajectory from the infinite number possible? In what coordinate frame is the trajectory planned? However, these complexities offer new research opportunities; investigations of multijoint movements may provide considerable insight into the strategies employed by the central nervous system in the control of skilled activities.

Recently, a few studies of the kinematic and dynamic aspects of multijoint human and monkey arm movements have been conducted. The objective of these studies was to identify common kinematic features or stereotyped patterns of muscle activation characterizing these movements (Georgopoulos et al., 1981; Soechting and Lacquaniti, 1981; Morasso, 1981; Abend et al., 1982; Hollerbach and Flash, 1982). The planning and control of the kinematic aspects of arm movements is termed trajectory formation. The term trajectory refers to the configuration of the arm in space and to the speed of movement as the hand moves from its initial to its final position. Some investigators (Greene, 1972; Saltzman, 1979; Soechting and Lacquaniti, 1981) have argued that trajectories are planned in joint variables. It has been claimed that the CNS uses a strategy of maintaining constant ratios between angular velocities of the joints in order to bring about a reduction in the complexity of the control problem by reducing the number of degrees of freedom. In contrast to this view, other investigators have argued that simplicity of motor control is achieved by planning hand trajectories in extracorporal space; joint rotations are then tailored to produce these desired hand movements (Lashley, 1951; Bernstein, 1967). Recently, this view has gained support from studies of planar, unconstrained human and monkey movements (Georgopoulos, 1981; Morasso, 1981; Abend et al., 1982). When moving the hand between pairs of targets, subjects tended to generate roughly straight hand trajectories with single-peaked, bell-shaped speed profiles; this behavior was independent of the part of the work-space in which the movement was performed. Because the common invariant featurcs of these movements were only evident in the extracorporal coordinates of the hand, these results are a strong indication that planning takes place in terms of hand trajectories rather than joint rotations.

Can this conclusion be generalized to more complex movements? When subjects were instructed to generate curved movements, the single-peaked hand speed profile was not preserved. Although the 
hand paths appeared smooth, their curvature 4 was not uniform, and the hand speed displayed one or more maxima. The minima between two adjacent speed peaks correspond temporally to peaks in the curvature (Abend et al., 1982). A similar temporal coupling between speed and curvature has been observed in handwriting, drawing (Viviani and Terzuolo, 1980), and infant reaching movements (von Hofsten, 1979). In this paper, we will show that this behavior can be derived from a single organizing principle. This principle is based on the kinematics of the motion of the hand in extracorporal space and provides further evidence that movements are planned in terms of hand trajectories rather than joint rotations.

To describe this behavior, a mathematical model of the organization of voluntary arm movements is presented. There are, of course, many ways of formulating a mathematical description of any given phenomenon. In the work presented here, we have used dynamic optimization theory, as it permits us to describe an assumed goal of this class of movements in a relatively simple formula and derive from the formula a detailed prediction of the kinematics of a large number of specific movements. It will be shown that this mathematical model succeeds in accounting for the majority of the kinematic features of planar horizontal arm movements described in previous studies (Morasso, 1981; Abend et al., 1982). A number of new features of planar horizontal arm movements are also predicted by the model, and some new experimental results confirming these predictions are presented.

\section{The Mathematical Model}

Briefly, dynamic optimization requires the definition of a criterion function which describes the objective of the movement. Generally, this function is expressed mathematically as a time integral of a performance index, an algebraic function which may, in general, depend on the system inputs, outputs, and internal variables. A set of differential equations are formulated which describe the response of the system to its inputs. The methods of variational calculus and optimal control theory (Bryson and Ho, 1975; Pontryagin et al., 1962) are applied to find the trajectory which minimizes this criterion function subject to dynamic constraints imposed by the system differential equations and the algebraic constraints imposed at the end points or during the motion. The use of optimization techniques to model natural behavior is appealing because of the analogy it bears to the optimization presumed to occur as a result of natural selection. Studies of two-joint arm movements have shown that as a result of practice the variability in hand trajectories is reduced exponentially with time (Georgopoulus et al., 1981). The fact that only a few executed trajectories emerge may indicate an underlying adaptive process tending to produce movements which optimize certain kinematic or dynamic variables (Abend et al., 1982). The critical step in the analysis is the selection of an appropriate objective function. Here the experinental results are suggestive. Since, with learning and practice, movements tend to be performed more smoothly and gracefully, this may indicate an underlying objective of achieving the smoothest movement which carries the hand from one equilibrium position to another. This was the point of departure of an optimization-based mathematical description of voluntary movements in monkeys (Hogan, 1982, 1984; Bizzi et al., 1984). The major qualitative and quantitative features of single-joint forearm movements have been successful predicted, assuming that maximizing smoothness may be equated to minimizing the mean-square jerk. Jerk is mathematically defined as the rate of change of acceleration. The work presented in this paper is the generalization of that analysis to the case of multijoint motion.

An important feature of the multijoint case is that the predicted

${ }^{4} \mathrm{H}$ and speed, $T$, is defined as: $T=\sqrt{(\dot{x})^{2}+(\dot{y})^{2}}$. Trajectory curvature is defined as: $C=(\dot{x} \bar{y}-\dot{y} \bar{x}) /\left((\dot{x})^{2}+(\dot{y})^{2}\right)^{3 / 2}$ where $\dot{x}$ and $y$ are the time derivatives of the $x$-and $y$-coordinates of the hand in the plane and $\ddot{x}$ and $\ddot{y}$ are the corresponding accelerations. behavior depends critically on the choice of coordinates in which the criterion function is formulated. Our choice of coordinates was again guided by experimental observations, in particular the fact that the invariant features of upper limb movements are orly evident when hand motion is expressed in extracorporal coordinates.

The position vector of the hand was defined with respect to a laboratory-fixed Cartesian coordinate system. Differentiating this position vector three times, Cartesian jerk for the hand can be defined. In moving from an initial to a final position in a given time $t_{f}$, the objective function to be minimized is the time integral of the square of the magnitude of jerk:

$$
C=\frac{1}{2} \int_{0}^{t_{f}}\left(\left(\frac{d^{3} x}{d t^{3}}\right)^{2}+\left(\frac{d^{3} y}{d t^{3}}\right)^{2}\right) d t
$$

$x$ and $y$ are the time-varying hand position coordinates. Mathematical expressions for $x(t)$ and $y(t)$ are to be found, which bring the criterion function in equation 1 to a minimum.

This optimization procedure was used for the description of several experimentally observed types of human planar two-joint arm movements: unconstrained point-to-point movements, unconstraincd curved movements, and obstacle-avoidance movements. Comparisons of the mathematically predicted trajectories with experimental movement records were used to evaluate the success of the model.

Unconstrained point-to-point movements. For unconstrained point-to-point movements, the objective can be stated as follows:

"Generate the smoothest motion to bring the hand from the initial position to the final position in a given time."

One might expect the physical system which generates the movement (i.e., the neuromusculoskeletal system) to impose certair constraints on the kinematic or dynamic variables. Such constraints might set limits on the hand speed or acceleration, due, for example, to limitations on the maximum torques that the system can generate or on how rapidly they can be changed (Nelson 1983). However, none of the movements studied here was extremely fast nor did any of the movements require the generation of large forces. As the subjects did not operate anywhere near the limits of neuromuscular performance these constraints are inoperative. The optimization procedure is presented in Appendix $A$ and results in a minimum jerk trajectory which is a fifth order polynomial in time for both $x(t)$ and $y(t)$.

If the constraints imposed by the physical system become important, the problem may be solved using the method of Pontryagin (Pontryagin et al. 1962). This method was also applied to our problem (Appendix B). The two methods yielded the same expressions for the hand trajectory.

The criterion function determines the form of the movement trajectory. The details are determined by the boundary conditions at the onset and termination of the movement. Given this information and the duration of the movement, the trajectory of the hand is specified in its entirety. No other information is required. Assuming the movement to start and end with zero velocity and acceleration, the following expressions for hand trajectory are obtained:

$$
\begin{aligned}
& x(t)=x_{0}+\left(x_{0}-x_{f}\right)\left(15 \tau^{4}-6 \tau^{5}-10 \tau^{3}\right) \\
& y(t)=y_{0}+\left(y_{0}-y_{f}\right)\left(15 \tau^{4}-6 \tau^{5}-10 \tau^{3}\right)
\end{aligned}
$$

where $\tau=t / t_{f}, x_{0}, y_{0}$ are the initial hand position coordinates at $t=$ 0 , and $x_{f}, y_{f}$ are the final hand position coordinates at $t=t_{f}$.

Profiles of the predicted hand path $(P)$ and hand tangential velocity $(T)$ are shown in Figure $1 A$. This trajectory is a straight line between initial and final positions with a bell-shaped unimodal velocity profile. Since the predicted trajectory depends only on the initial and final positions of the hand, it is invariant under translations and rotations. The shape of the predicted trajectory also does not change with amplitude or duration of the movement, which merely serve to change the scale of the position and time axes, respectively. Note 


\section{(A)}
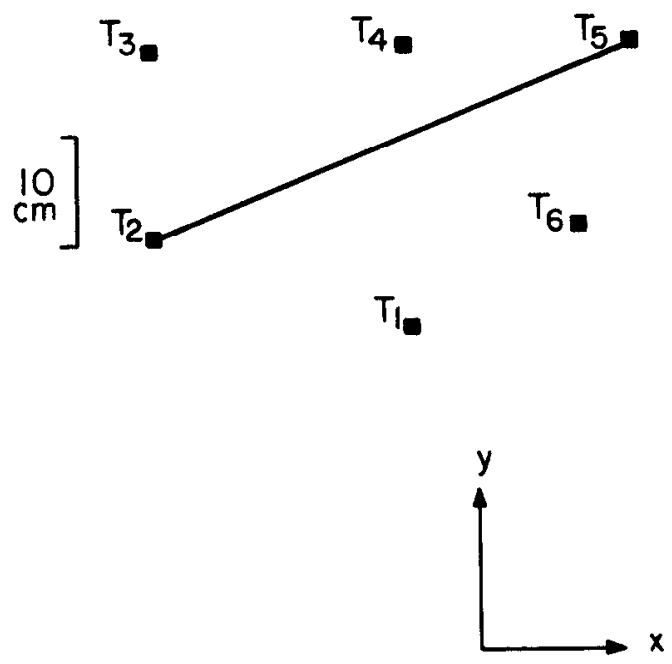

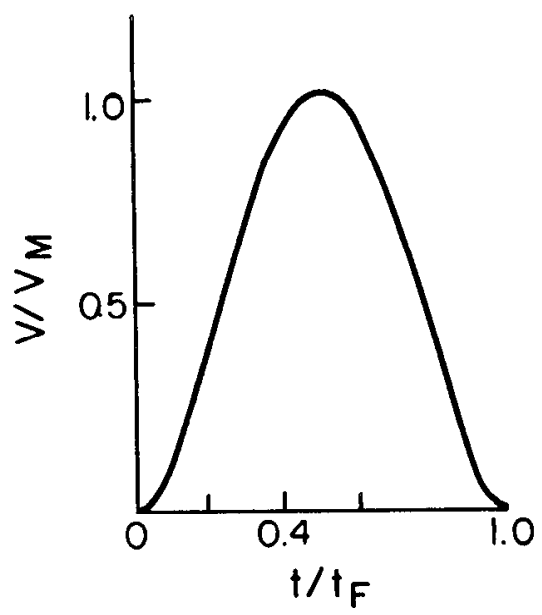

(B)
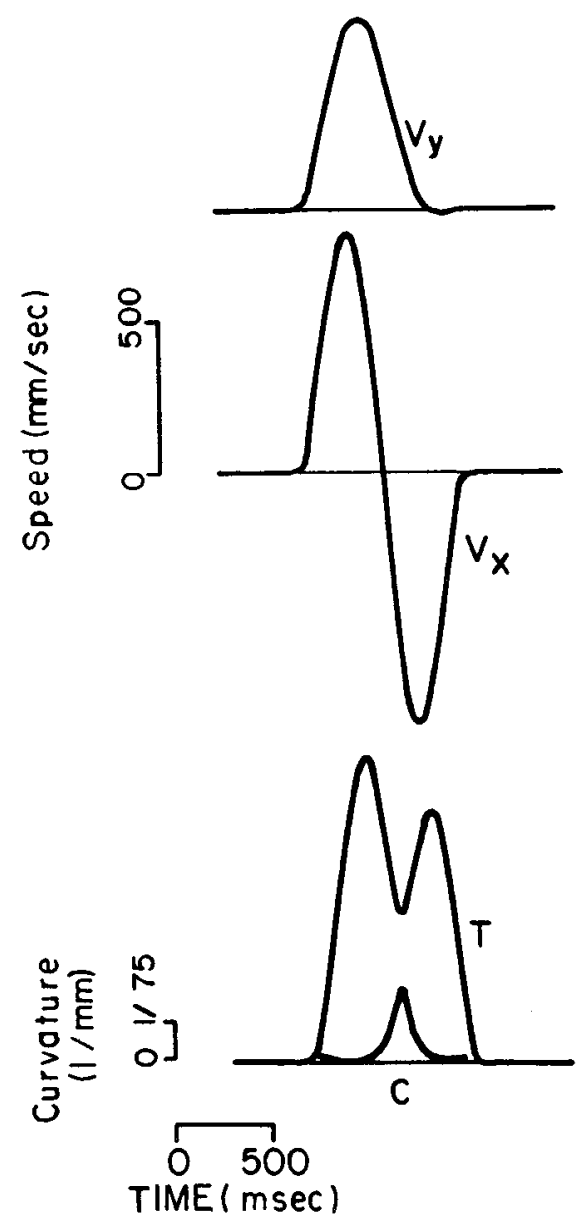

Model

Figure 1. A, A representative example of a predicted minimum-jerk unconstrained point-to-point hand trajectory, $T_{1}$ to $T_{6}$ represent the targets. The plot of the predicted tangential velocity profile illustrates the hand speed, $V$, relative to the maximal speed, $V_{M}$, as a function of $t / t_{f}$, where $t$ is the time and $t_{f}$ is the movement duration. $B, A$ representative example of a predicted curved minimum-jerk hand trajectory between targets 1 and 4 . The plot illustrates the predicted hand path, $P$, hand tangential velocity, $T$, hand curvature, $C$, and hand velocity components along the $x$-axis, $V_{x}$, and along the $y$ axis, $V_{y}$. The meanings of $d_{1}, d_{2}$, and $d_{3}$ are explained in the text. 
also that the expression describing the trajectory along the line joining the initial and final positions is a single fifth order polynomial in time which is identical to the minimum-jerk trajectory for singlejoint motion (Hogan, 1982, 1984).

Curved point-to-point movements. To model curved and obstacleavoidance movements, it was assumed that the hand is required, in its motion between the end points, to pass through a third specified point. Hence, for these movements, the movement objective can be stated as follows:

"Generate the smoothest motion to bring the hand from the initial position to the final position in a given time. The hand must move to the final position through a specified point (a via point) at an unspecified time."

The requirement that the hand should move through a specified via point defines equality constraints; i.e., the hand position is prescribed at some intermediate time, $t_{1}$, between the onset and the termination of the movement. Hence, if the location of the via point with respect to a Cartesian coordinate system is given by the coordinates $\left(x_{1}\right.$ $\left.y_{1}\right)$, the equality constraints on the hand position coordinates, $x(t)$ and $y(t)$, at time $t_{1}$, are

$$
\begin{aligned}
& x\left(t_{1}\right)=x_{1} \\
& y\left(t_{1}\right)=y_{1}
\end{aligned}
$$

The time $t_{1}$ at which the hand has to pass through this point is not a priori specified but is derived from the optimization procedure. It is one of the predictions of the mathematical model. Problems of this kind are known as dynamic optimization problems with interior point equality constraints, and techniques have been established for their solution (Bryson and Ho, 1975). The optimal trajectory for the entire movement between the initial and final position is derived in appendix $\mathrm{C}$. For the particular problem treated here, the technique used requires the continuity of the velocity and acceleration at this intermediate time. Since discontinuities of hand velocities and accelerations would require infinite accelerations and jerks, this is a reasonable requirement for arm movement. The details of the predicted movements, including the time at which the hand passes through the via point, depend on the chosen boundary conditions at time $t=0$ and $t=t_{i}$ and on the specified interior point.

Applying the optimization technique, one obtains an expression for the position component $x(t)$ at all times $t \leq t_{1}$ :

$$
\begin{aligned}
x^{-}(\tau)= & \frac{t_{f}^{5}}{720}\left(\pi _ { 1 } \left(\tau_{1}^{4}\left(15 \tau^{4}-30 \tau^{3}\right)\right.\right. \\
& \left.+\tau_{1}^{3}\left(80 \tau^{3}-30 \tau^{4}\right)-60 \tau^{3} \tau_{1}^{2}+30 \tau^{4} \tau_{1}-6 \tau^{5}\right) \\
& \left.+c_{1}\left(15 \tau^{4}-10 \tau^{3}-6 \tau^{5}\right)\right)+x_{0}
\end{aligned}
$$

and for times $t \geq t_{1}$ the expression is:

$$
\begin{aligned}
x^{+}(\tau)= & \frac{t_{f}^{5}}{720}\left(\pi _ { 1 } \left(\tau_{1}^{4}\left(15 \tau^{4}-30 \tau^{3}+30 \tau-15\right)\right.\right. \\
& \left.+\tau_{1}^{3}\left(-30 \tau^{4}+80 \tau^{3}-60 \tau^{2}+10\right)\right) \\
& \left.+c_{1}\left(-6 \tau^{5}+15 \tau^{4}-10 \tau^{3}+1\right)\right)+x_{f} \\
& =x^{-}(\tau)+\pi_{1} \frac{t_{f}^{5}(\tau-\tau 1)^{5}}{120}
\end{aligned}
$$

These equations depend on the dimensionless variables $\tau_{1}=t_{1} / t_{f}$ and $\tau=t / t_{f}$. Similar expressions are obtained for $y^{+}(\tau)$ and for $y^{-}(\tau)$ with constant coefficients, $\pi_{2}, c_{2}$ replacing $\pi_{1}$ and $c_{1}$ in equations 4 and 5 . These four coefficients, $\pi_{1}, c_{1}, \pi_{2}$, and $c_{2}$ which depend on the position coordinates at the boundaries and at the interior point and on $t_{1}$ are defined in Appendix $\mathrm{C}$

A typical predicted minimum-jerk curved movement is shown in Figure $1 B$. Displayed are the hand path $(P)$ and the hand tangential velocity $(T)$ and curvature $(C)$ profiles. In addition, the two velocity profiles along the $x$-axis, $V_{x}$, and the $y$-axis, $V_{y}$, are also shown in Figure 1B. As this figure dernonstrates, the predicted harnd curvature increases reaches a maximum, and then decreases again as the movement approaches the final position. The tangential velocity curve exhibits two peaks, and the minimum in the tangential velocity curve occurs at the same time as the maximum curvature point. Again, the predicted trajectories are invariant under translation, rotation, time scaling, and amplitude scaling.

\section{Experimental Methods}

To evaluate the model, simulated trajectories were compared to measured hand trajectories. Planar horizontal arm movements were recorded, using the apparatus shown in Figure 2. The subject was seated and held the handle of a two-link mechanical manipulandum. The shoulder was restrained throughout these experiments. The subject's wrist was braced in some of the trials; in others it was free to move. The subject was instructed to move the handle of the manipulandum under targets mounted on a horizontal plexiglass panel above the apparatus, upon their illumination. Visual information about the arm location was eliminated by darkening the room in which recordings took place. The joint angles of the mechanical apparatıs were monitored by means of precision potentiometers. The potentiometer voltage signals were digitized at a rate of 100 samples/sec and stored on magnetic tapes. These signals were filtered with an upper cut-off frequency of $5.2 \mathrm{~Hz}$. A calibration procedure, executed before each experiment, determined the parameters for the conversion of these voltage signals to apparatus joint angles. Off-line data analysis was performed to compute the subject's hand position, and, based on the measured geometry of the subject's upper extremity, joint angles were also computed. Lagrange polynomial differentiation was used to obtain joint angular velocities, hand velocities, and the curvature of the hand path. Handle movements as small as $1.0 \mathrm{~mm}$ could be detected, and the error in the computed hand velocity was less than $4 \%$. The error in computed hand curvature was less than $6 \%$.

This apparatus and procedure are the same as used by Abend et al. (1982), and in this paper their results are compared to predictions of the mathematical model. The model also predicts several additional features of movement and, to test these predictions, new experiments were performed. Abend et al. used four experimental paradigms. In their first paradigm, the subjects were instructed to move the hand from one target to another upon illumination of the second target. No instructions were given regarding the path between the two targets. We used the same experimental paradigm but instructed the subject to move at various speeds as follows: the subject was told that the target LED would first be illuminated for a duration equal to the desired movement duration (between 0.5 and $1.0 \mathrm{sec}$ ), then turned off for a brief period ( $100 \mathrm{msec}$ ), and then turned on again. The subject was to move when the target was illuminated for the second time. No specific instructions were given with regard to accuracy of the movement. No feedback of results was given.

In the second paradigm used by Abend et al. (1982), subjects were instructed to move the hand to a target along a self-generated curved path.

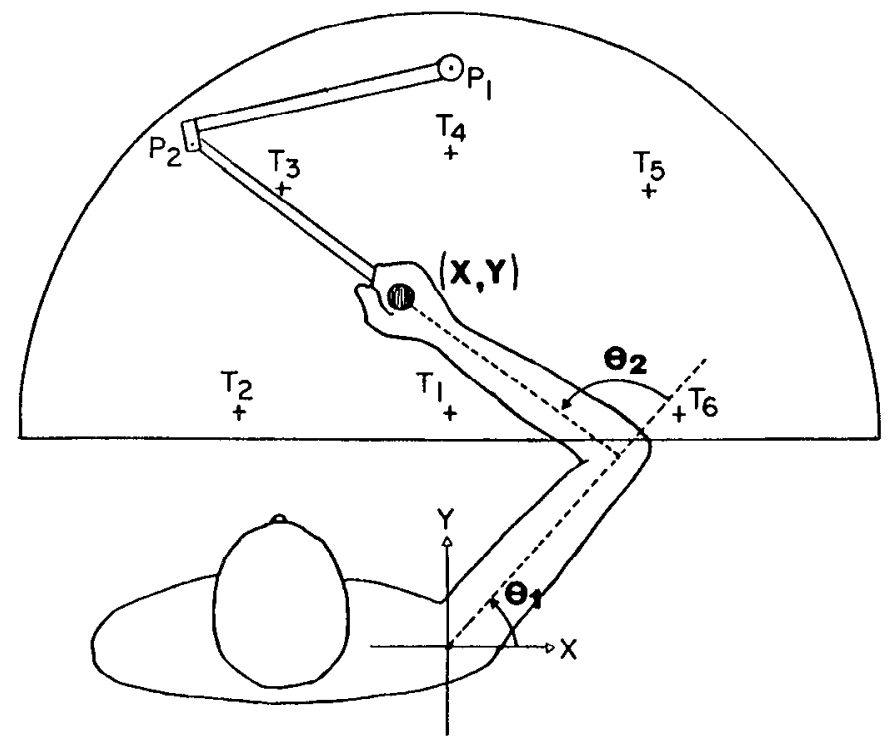

Figure 2. Experimental apparatus for measuring arm trajectories in a horizontal plane. $T_{1}$ to $T_{6}$ are the LED targets. $\theta_{1}$ and $\theta_{2}$ are, respectively, the subject's shoulder and elbow joint angles. Movement of the handle was measured by way of the potentiometers, $P 1$ and $P 2$. 
No guide or obstacle was presented. In their third group of experiments subjects were instructed to follow curved guides. These were constant curvature arcs placed on top of the plexiglass panel. Their fourth paradigm requircd obstacle avoidance. Subjects were instructed to move their hand from one target to another while avoiding an "obstacle" represented by a row of red light-emitting diodes. The maximum length of obstacles used in their experiments was $25 \mathrm{~cm}$. The obstacle was placed belween the targets with its long axis perpendicular to the line connecting the two targets.

In the above paradigms, the subjects were not constrained to move the hand through any specific intermediate point on route to the target. We were therefore interested in determining what effect the introduction of a real via point would have on the movement. Experiments were conducted by us in which subjects were instructed to generate continuous movements from one target to another through an intermediate target. The intermediate target was lit throughout the movement. Movements involving the same set of three targets were performed at the subject's preferred speed first and then at a faster speed. Overall data obtained from nine subjects are presented in this paper.

In most experiments, the movements were confined to elbow and shoulder rotations in a horizontal plane at the subject's shoulder level. In some experiments, however, wrist movements were also allowed and/or subjects were instructed to move their arm in a horizontal plane passing through their waist.

To determine model-predicted trajectories for comparison, the values of several kinematic variables were derived from the experimental data. For straight trajectories, these values were the initial and final target locations and the movement durations. For unconstrained curved and obstacle avoidance movements, the location of the via point also had to be specified. This was determined from the location of the maximum curvature point. In experiments with an intermediate target, the location of the via point was taken to be the location of this target. These parameters were substituted in the polynomials derived from the dynamic optimization, and hand positions were computed every $10 \mathrm{msec}$. These positions were then differentiated to derive hand velocities and differentiated again to derive hand accelerations and the curvature was computed.

\section{Results}

Straight movements. Typical experimental results for two uncon strained point-to-point movements are shown in Figure 3 superim posed on the predicted minimum-jerk trajectories for the same movements. As this figure shows, there is a good qualitative and quantitative match between the predicted and actual trajectories. There is a good agreement between the predicted and measured steepness of the rising and falling parts of the tangential velocity and acceleration curves and the time at which maximum acceleration is reached. The predicted trajectory does not capture the asymmetry in the tangential velocity profile of the measured trajectory. The difference, however, is quite small. There are also some slight discrepancies between the measured and predicted acceleration profiles.

We tested the predictive capability of the minimum-jerk model as follows. For minimum jcrk unconstraincd point to-point trajectories, the following relation between the maximum hand speed $\left(V_{\max }\right)$, and the ratio of movement amplitude $(A)$ to movement duration $\left(t_{f}\right)$ may be derived: $V_{\max }=C A / t_{i}$, where $C=1.875$. The mean value of $C$ derived from 30 measured unconstrained point-to-point movements, is 1.805; therefore the mean error in $C$ is $3.7 \%$ of its predicted value. The standard deviation of $C$ is $0.153,8.2 \%$ of its predicted value. Given that our velocity measurements were accurate to $4 \%$ (see "Experimental Methods"), this result indicates that model and observations agree within experimental error. We tested the hypothesis that $C=1.875$ using the Student's $t$ test. It was accepted at the 0.01 level but rejected at the 0.05 level

Typical examples of the fit between the temporal behavior of real and simulated shoulder and elbow angles and angular velocities are shown in Figure 4. To obtain joint angles and angular velocities, the inverse kinematics problem (Brady et al., 1982) was solved. Since, for horizontal planar two-joint movements, hand trajectories uniquely define angular trajectories, this computation serves only to permit an additional comparison between the theoretical and experimental results. As can be seen in Figure 4, the actual and predicted angular velocity profiles agree quite well, and there exist only minor discrepancies between the simulated and real trajectories. Note also the lack of invariance of joint trajectories to translation and rotation of the movements compared to the invariance of hand paths and speed profiles.

Curved movements. A typical example for a predicted curved trajectory was shown above in Figure $1 B$. As this example demonstrates, the predicted trajectories display the majority of the observed features of real curved hand trajectories as discussed by Abend et al. (1982). If real curved hand trajectories can be described adequately by the proposed model, they should display the kinematic features exhibited by the simulated trajectories. To facilitate the explanation of the nature of these predictions, a simple geometric construction, shown in Figure $1 B$, is used. The movement end-points are connected by a straight line. The perpendicular to this line which passes through the via point is drawn. This second line, of length $d_{3}$, divides the line connecting the two movement end-points into two segments of lengths $d_{1}$ and $d_{2}$.

The first predicted kinematic leature relales to the dependency of the shape of the hand velocity and curvature profiles on the location of the via point. If the via point is displaced in any direction, towards either the initial or the final target, so that one of the two segments $d_{1}$ or $d_{2}$ is longer, the amplitude of the velocity peak on the corresponding portion of the movement will be higher.

The second predicted kinematic feature relates to the depth of velocity valley and the height of the curvature peak. For more highly curved movements, the tangential velocity dip is more pronounced. Hence, for two movements of the same duration with the same values of $d_{1}$ and $d_{2}$, the amplitude of the hand curvature peak will be higher, and the tangential velocity valley will be deeper for the movement with the larger value of $d_{3}$.

To test the validity of these two predictions, quantitatively simulated and measured hand trajectories were compared for movements through intermcdiate targets, unconstrained curved movements, and obstacle-avoidance movements. In all of the following figures, measured hand paths $(P)$, hand speed profiles $(T)$, hand curvature profiles $(C)$, and the profiles of the two velocity components along the two orthogonal axes $\left(V_{x}\right.$ and $\left.V_{y}\right)$ are displayed in the left column (Real) and the corresponding plots for the minimum jerk trajectories are displayed in the right column (Model).

Comparisons of predicted and measured hand trajectories for arm movements generated by subjects in experiments with intermediate targets are shown in Figures 5 to 7 . Figures 5 and 6 describe results from trials in which the location of the via point, with respect to the initial and final targets, required the reversal of the direction of the hand movement along the $y$-axis. In this group of movements, the intermediate target was tirst placed at equal distances from the initial and final targets, closer to (Fig. 5A) or further away (Fig. 5B) from the line connecting these two targets, and then was symmetrically offset from the center in both directions along a line passing parallel to the $x$-axis (Fig. $6, A$ and $B$ ). As these figures show, there is a good qualitative and quantitative tit between the measured and predicted trajectories. Furthermore, the measured hand trajectories validated the above predictions. Figure 7 shows results from trials in which the locations of the intermediate targets required a reversal of movement direction along both the $x$ - and $y$ axes. As this figure demonstrates, the fit of the predicted to the real trajectories was equally good under conditions of both translation and rotation of the movements.

Two obstacle-avoidance movements are compared to the corresponding simulated trajectories in Figure 8. Two unconstrained curved movements are compared to simulated trajectories in Figure 9. Again all of the above predicted features are exhibited. Note also the similarities in the kinematic characteristics of obstacle avoidance movements, unconstrained movements, and movements through intermediate targets.

Additional predictions can be made from the model. One is similar to the "isochrony principle" (Viviani and Terzuolo, 1982). In the 
a
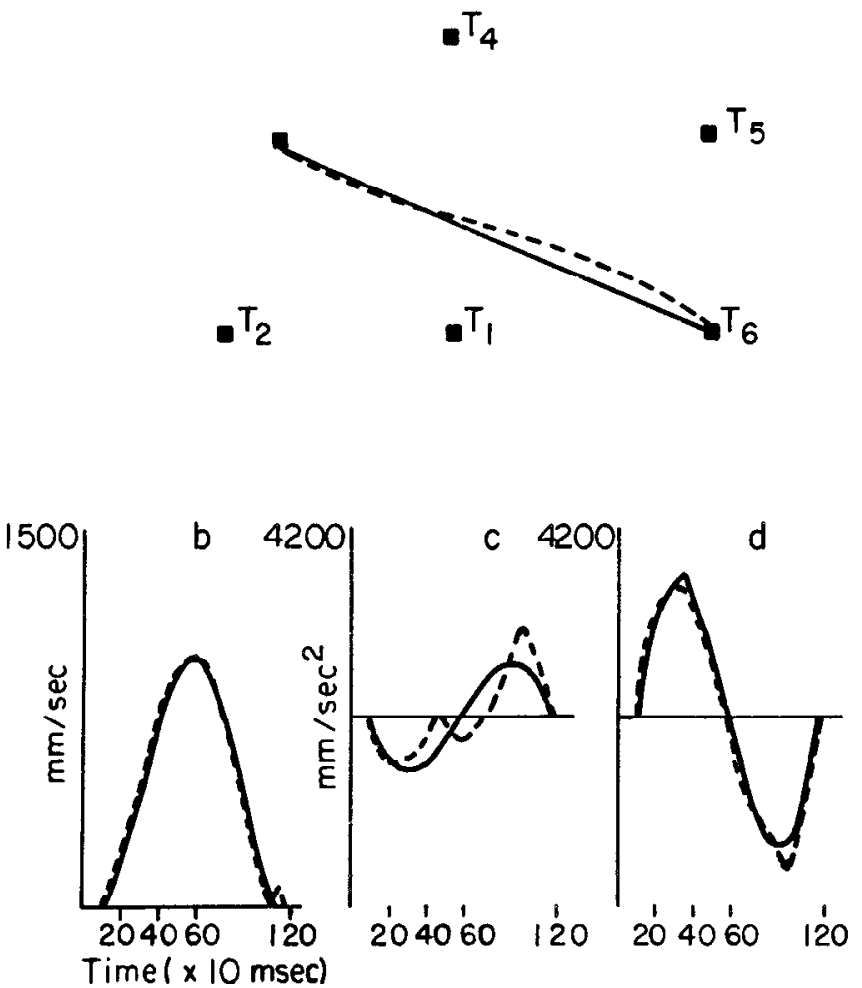

A a

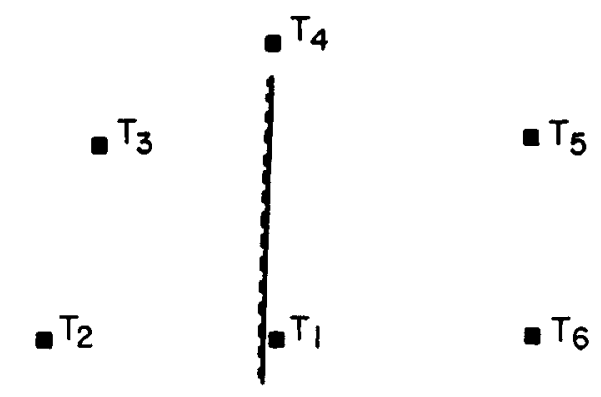

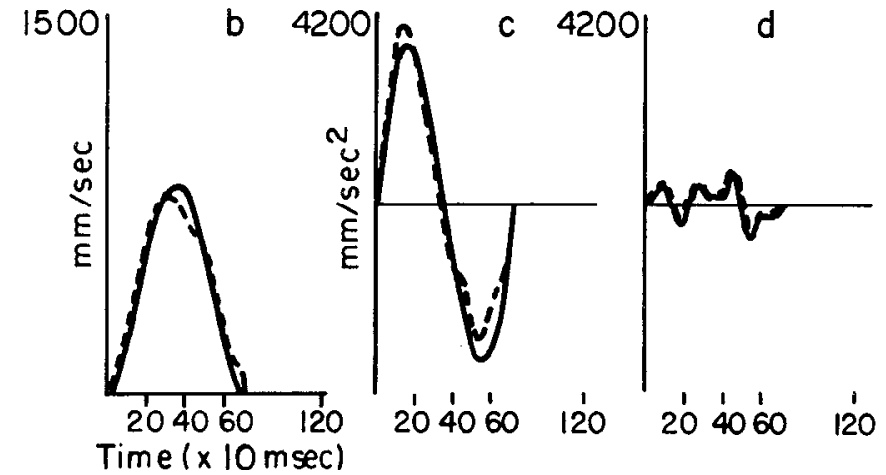

B

Figure 3. Overlapped predicted (solid lines) and measured (dashed lines) hand paths (a), speeds (b), and acceleration components along the $y$-axis (c),

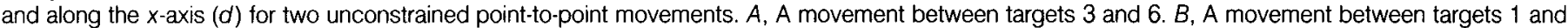
4 .

context of this work, the isochrony principle would state that the times it takes the hand to move along the two portions of the movement, from the start to the via point $\left(t_{1}\right)$ and from the via point to the end of the movement $\left(t_{2}\right)$ should be approximately equal. Our model gives a more explicit, numerically testable prediction stating that the $t_{1} /\left(t_{1}+t_{2}\right)$ values should be roughly equal to 0.5 for most of $d_{1} /\left(d_{1}+d_{2}\right)$ values between 0 and 1 , except for cases in which the via point is very close to either one of the movement end-points. It is also predicted that for similar values of $d_{1} /\left(d_{1}+d_{2}\right)$, the values of $t_{1} /\left(t_{1}+t_{2}\right)$ will be independent of $d_{3}$. This behavior of the minimumjerk trajectories is consistent with the differences in heights of the two velocity peaks on either sides of the velocity valley because, if the hand travels along both movement segments in roughly the same time, then the velocity amplitude for the longer segment should be higher.

In the following figures, the measured $t_{1} /\left(t_{1}+t_{2}\right)$ values were derived from unconstrained curved movements and from movements through intermediate targets for which the via-points of the predicted trajectories coincided with the maximum curvature points of the measured movements. In Figure $10 A$, points corresponding to 60 measured $t_{1} /\left(t_{1}+t_{2}\right)$ values for various experimentally set $d_{1} /$ $\left(d_{1}+d_{2}\right)$ parameters are superimposed on the predicted $t_{1} /\left(t_{1}+t_{2}\right)$ versus $d_{1} /\left(d_{1}+d_{2}\right)$ curve. A plot of the residual error between predicted and measured values of $t_{1} /\left(t_{1}+t_{2}\right)$ versus $d_{1} /\left(d_{1}+d_{2}\right)$ is shown in Figure $10 B$. The residual error shows no trend. The mean error is -0.004 , less than $1 \%$ of the typical value of $t_{1} /\left(t_{1}+t_{2}\right)(0.5)$. The standard deviation of the error is $0.02,4 \%$ of the typical value of $t_{1} /\left(t_{1}+t_{2}\right)$. The hypothesis that the mean error was zero was accepted on the basis of the Student's $t$ test at the $0.001,0.05$, and
0.01 levels. These results show that within experimental error, the model is in excellent agreement with the observations.

Similarly, in Figure $10 \mathrm{C}$, points corresponding to 22 measured $t_{1} /$ $\left(t_{1}+t_{2}\right)$ versus $d_{3} /\left(\operatorname{maxd}_{3}\right)$ for various movements wilh similar values of $d_{1} /\left(d_{1}+d_{2}\right)$ are superimposed on the corresponding predicted curve. A plot of the residual error between predicted and measured values of $t_{1} /\left(t_{1}+t_{2}\right)$ versus $d_{3} /\left(\max _{3}\right)$ is shown in Figure 10D. The mean error is -0.0006 , less than $0.2 \%$ of the typical value of $t_{1} /\left(t_{1}\right.$ $+t_{2}$ ) (0.49). The standard deviation of the error is $0.017,3.5 \%$ of the typical value of $t_{1} /\left(t_{1}+t_{2}\right)$. The hypothesis that the mean error was zero was accepted on the basis of the Student's $t$ test at the $0.001,0.05$ and 0.01 levels. Again the agreement between model and observations is excellent.

Another prediction of the model is that curved trajectories, like the straight trajectories, scale with time as the movement duration changes. Therefore, the path of the hand should be the same for fast and slow movements, while the hand speed throughout the movement should scale numerically by the ratio between the fast and slow movement durations. The scaling prediction is validated by the measured movements as demonstrated in Figure $11 A$, in which the movement duration is $1.1 \mathrm{sec}$ versus Figure $11 \mathrm{~B}$, with a movement duration of $0.85 \mathrm{sec}$. Moreover, as can be seen from this figure, the fact that the subjects move faster or slower does not affect the model performance.

The model predicts the same hand trajectories regardless of the specific joints involved in the generation of the movement or whether they are generated in a horizontal plane at the level of the subject shoulder or at other levels. Abend et al. (1982) presented experimental data obtained from trials in which the hand brace was removed allowing rotations at the wrist joint and trials in which the 
Figure 4. Measured and predicted shoulder, $S$, and elbow, $E$, joint angles and angular velocity profiles. A, A movement between targets 3 and $6 . B, A$ movement between targets 2 and 5 .
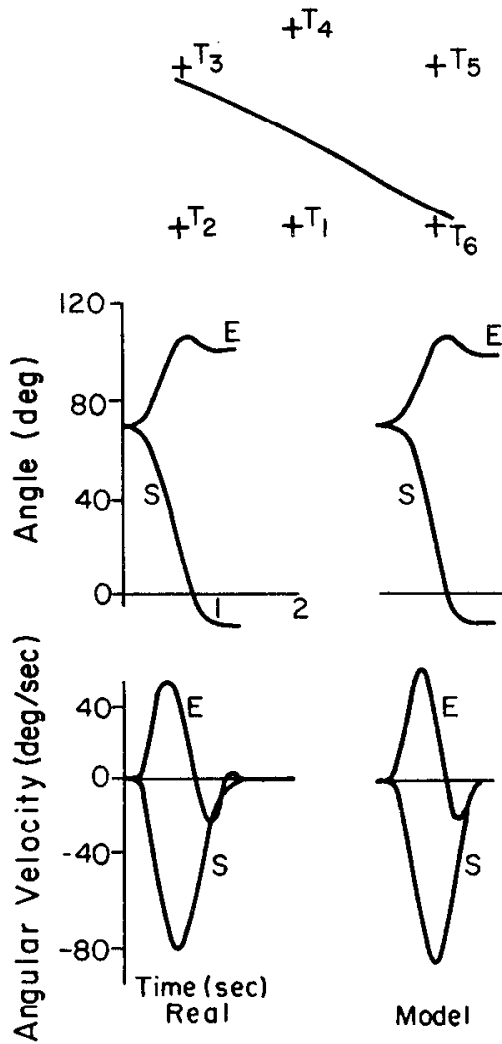

A
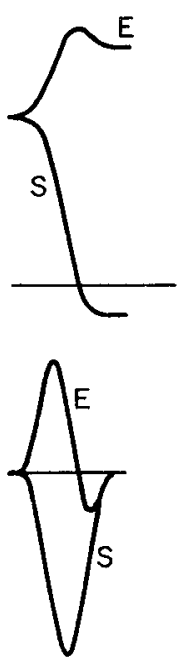

Model
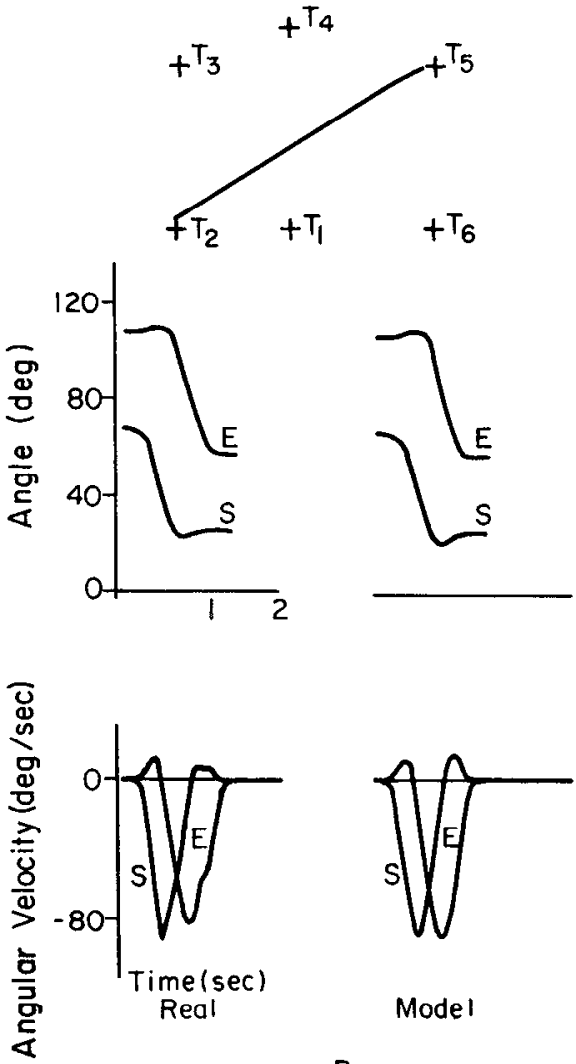

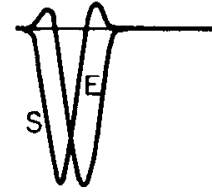

Mode I

B

movements were generated in a horizontal plane at the level of the subject's waist. The experimental apparatus used in our experiments and those of Abend et al. (1982) did not permit us to measure the exact trajectories of joint angles under these conditions, but as predicted by our model, the trajectories of the hand were no different from those described in the above figures.

The model succeeds in accounting for the majority of the kinematic features of the real trajectories. A few fine-grained details of the real movements, however, were not captured in the simulated trajectories. These include, for example, the tendency of the first velocity peak to be higher than predicted, in movements between targets 1 and 4 . Another example is the existence of irregularities in the hand path (the little "hooks") and hand speed as the hand approaches the goal target (e.g., Figs. 7 and 8).

\section{Discussion}

The mathematical model presented in this paper matches observed human planar two-joint arm movements. The organization of the movements was modeled through the criterion function. From the optimization of the criterion function, explicit analytic expressions for the description of many different hand trajectories were derived. The derivation of these expressions depended on the specification of a small number of parameters; in the case of unconstrained pointto-point movements, only the movement duration and the position, velocity, and acceleration at the endpoints were required. Application of the same model to curved movements required only the addition of the position coordinates of a via point. In return, the model yielded the time at which the hand passes through the via point and a detailed time-history of the hand positions, velocities etc. The mathematical analysis, particularly the dynamic optimization methods used, bring about a dramatic reduction in the dimensionality of the problem of describing movement kinematics.

The mathematical model presented in this paper cannot be dismissed as merely elaborate curve fitting, as it leads to several testable predictions: invariance of hand trajectories under translation and rotation in the workspace, scaling of hand trajectories with time and amplitude, temporal coupling between curvature and speed, and a prediction similar to the "isochrony principle." All of these kinematic characteristics have been observed in the experiments reported in this paper and in the motor control literature. For example, it has repeatedly been observed that peak velocity increases as an approximately linear function of the distance to be traveled, so as to keep the duration of the movement roughly constant (Viviani and Terzuolo, 1982). Temporal coupling between hand curvature and speed has been described previously (Viviani and Terzuolo, 1980; Abend et al., 1982), and movement scaling with time has also been observed for many different movements, including two-joint arm movements, three-dimensional reaching movements, handwriting, wrist movements etc. (Schmidt, 1980).

How does the mathematical model presented here compare with alternative descriptions? Only a few models for the mechanisms underlying trajectory formation have been proposed. Given the tendency to generate roughly straight hand paths in point-to-point movements and the phenomenon of the temporal coupling of hand curvature and speed, Abend et al. (1982) and Viviani and Terzuolo (1982) have suggested that this phenomenon is duc to a central mechanism which plans trajectories as sequences of movement segments which are then overlapped in time. Viviani and Terzuolo (1982) claim that movements obey an "isogony principle;" the ratio between tangential velocity and radius of curvature is piecewise constant. Our analysis of arm movements did not support this finding. Morasso and Mussa-Ivaldi (1982) suggest that curved movements are generated from separate strokes, each stroke characterized by various geometric parameters, such as length, total angular change, etc. Different movements may then be composed from different strokes, with different geometric parameters. Their model implies a need for storage and retrieval of strokes from a dictionary in memory; this dictionary would have to be very large.

These descriptions, however, do not make clear what rules and principles govern the choice of kinematic variables, nor why there is a tendency to generate straight paths in unconstrained movements in the first place. None of these descriptions of curved movements 

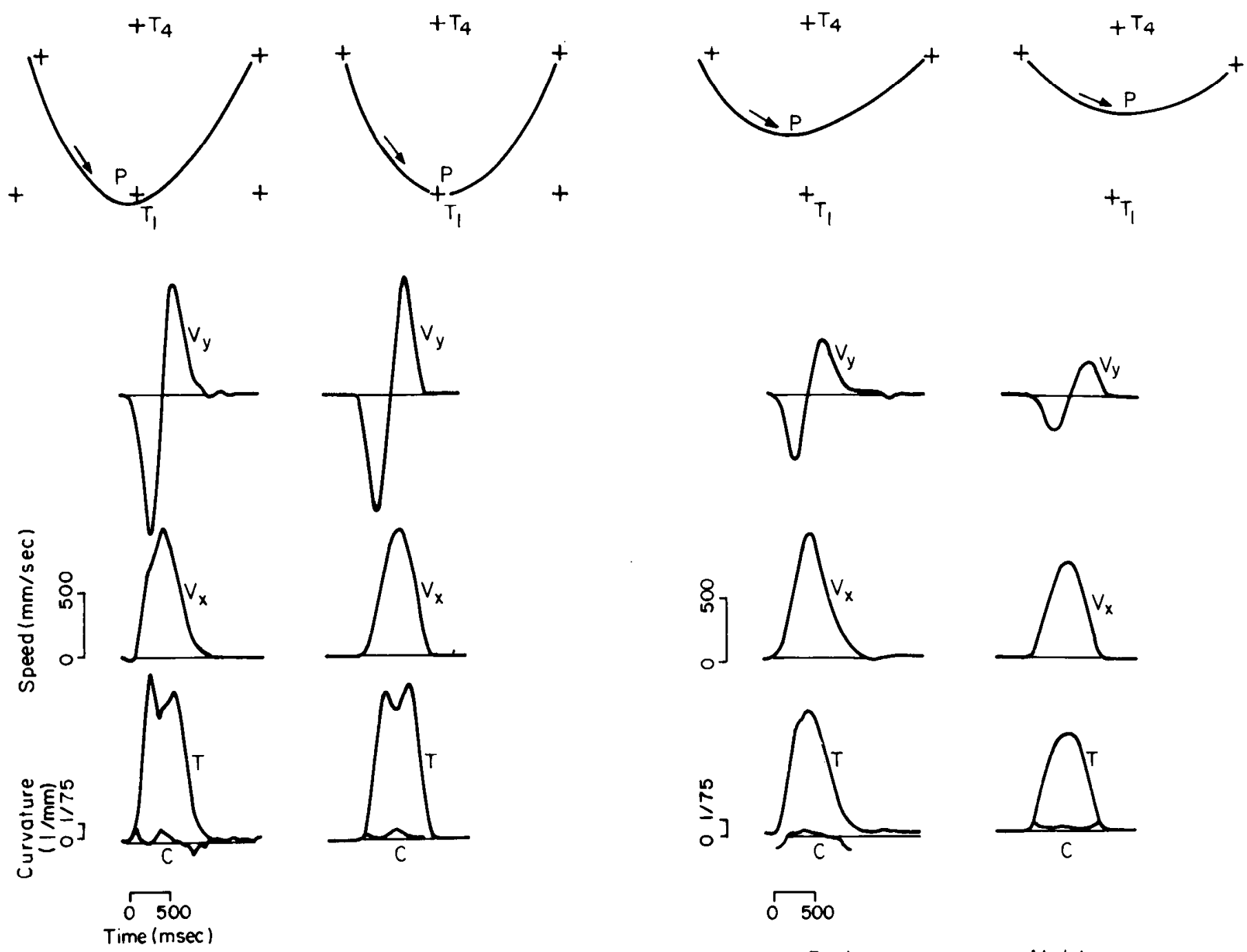

Real

Model

Real

\section{B}

Model

Figure 5. Representative examples of comparison between measured (Real, left columns) and predicted (Model, right columns) trajectories from a "viapoint" experiment. Displayed are the hand paths, $P$, and plots of hand speed, $T$, curvature, $C$, and velocity components, $V_{x}$ and $V_{y}$, versus time. The movement reversed its direction along the $y$-axis. $A$, I he intermediate target was located at equal distances trom the initial and final targets. $B$, The intermediate target was at equal distances from the movement end-points but closer to the line connecting them.

as smooth transition between separate strokes clarify what procedure is used to overlap succeeding strokes in time, nor do they propose any explanation for the isochrony principle. By contrast, based on dynamic optimization of a single criterion function, the model presented here accounted for the entire hand trajectory in both unconstrained and curved point-to-point movements. Furthermore, this model could be extended to describe more complicated movements by using more via points. However, a segmentation mechanism in the execution of hand trajectories is not ruled out by the optimization-based model, and it is possible that such a mechanism underlies the planning of long and complicated sequences of movement. Whatever the planning process, the work presented here makes it clear that not every inflection point in the hand path nor every velocity valley necessarily implies the beginning of a new stroke. The success of the model presented here indicates that a more basic principle than mere segmentation underlies the specification of hand trajectories. Some evidence in support of this suggestion can be found in Wing's (1978) studies of handwriting, in which it was shown that there exists a positive temporal correlation between the up and down strokes in the letter $n$, for example. This may indicate that an up-down stroke is planned as a single unit rather than two separate strokes.

This work also offers a plausible algorithm for Cartesian endeffector trajectory planning for artificial manipulators. Current methods of trajectory planning in robotics usually constrain the endeffector to follow straight lines using first-order polynomials (Taylor, 1979). For more complicated movements or movements through via points, end-effector trajectories are planned as series of simple second-order polynomials which are smoothly joined together (Brady, 1983) at knot points. While this method explicitly plans endeffector trajectories and assures that the end point will stay within the workspace, it does not guarantee low values of jerk. By contrast, the jerk minimization procedure presented here provides a rigorous way of planning trajectories of motions between equilibrium points and motions through via points while guaranteeing predictable and well-behaved trajectories.

The results presented here are independent of the physical system which generates the motion. No representation of the arm dynamics 

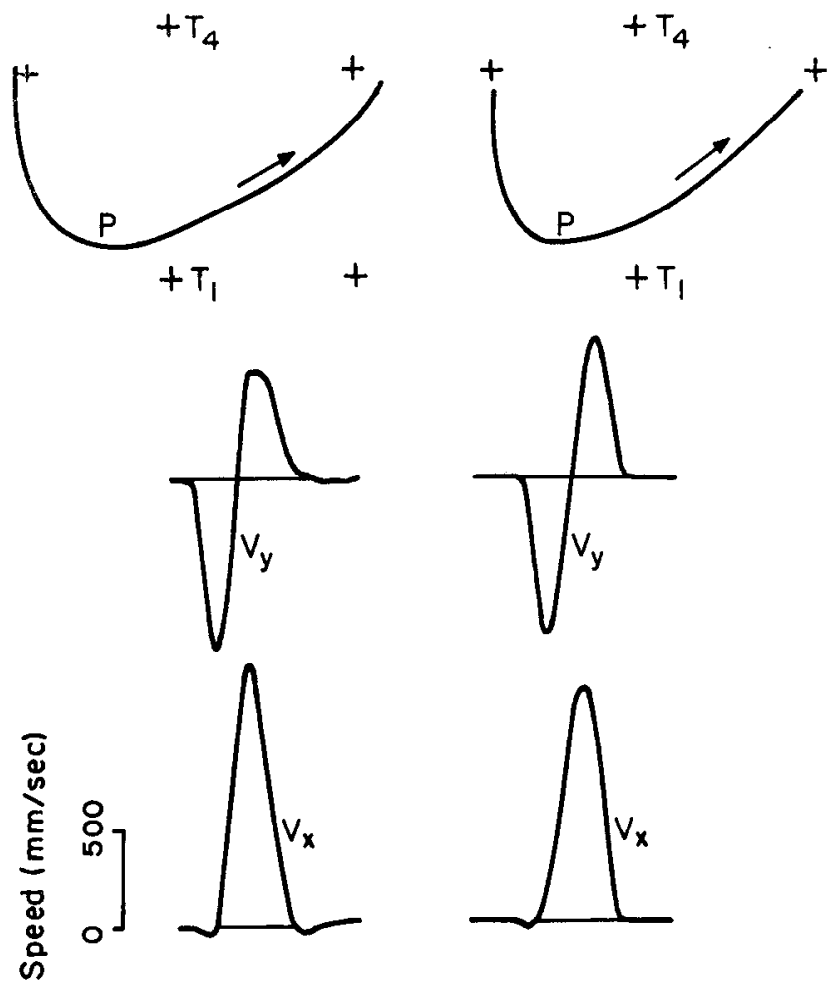

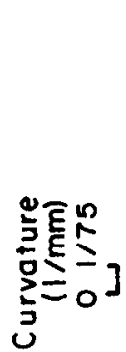
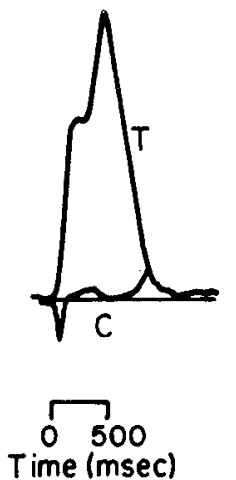

Real
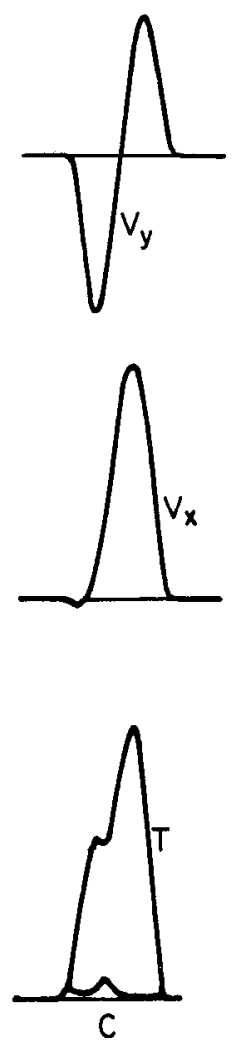

Model
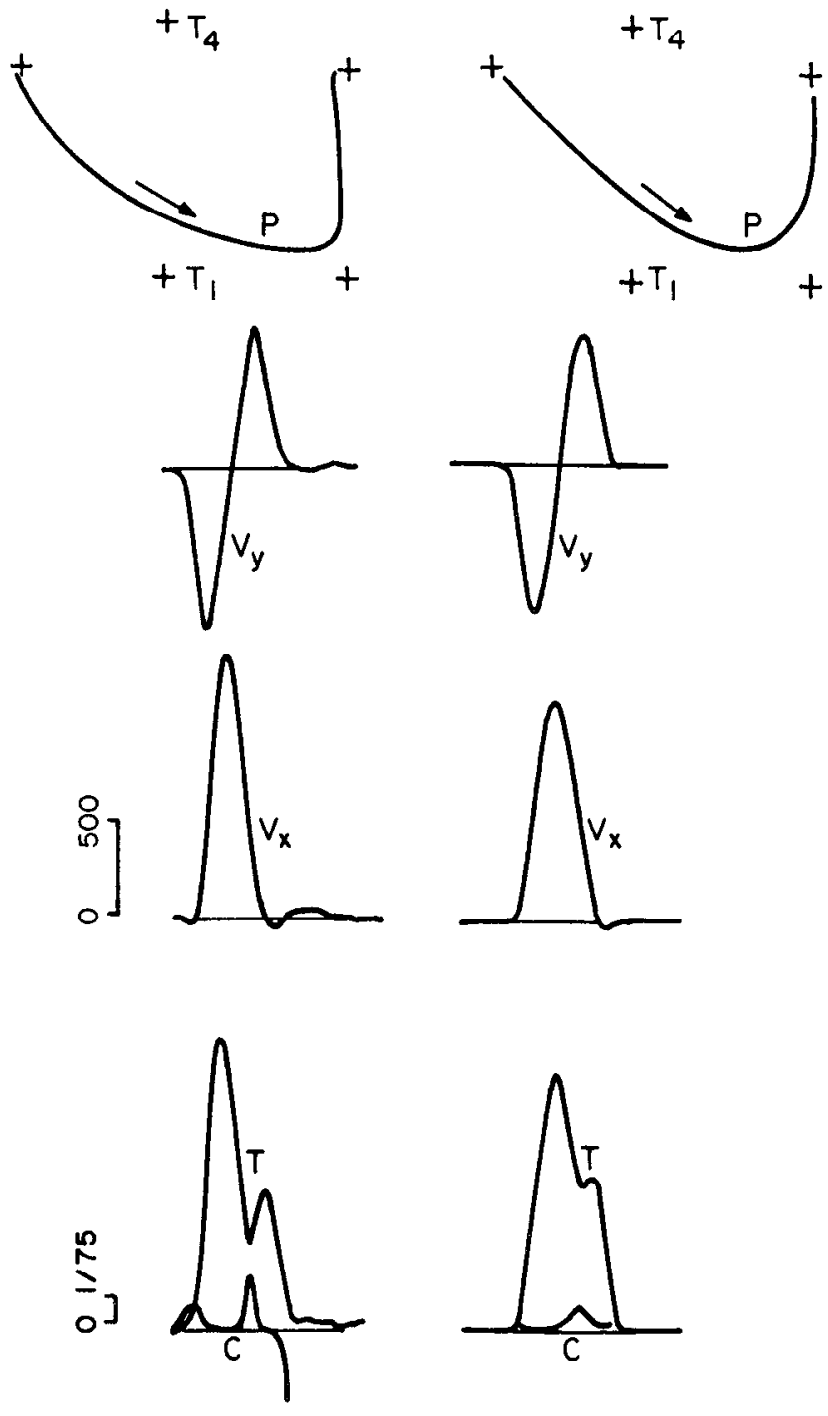

$\longdiv { 5 0 0 }$

Real

Model

\section{B}

Figure 6. Representative examples of comparisons between measured (Real, left columns) and predicted (Model, right columns) trajectories from a "via-point" experiment in which the movement reversed its direction along the $y$-axis. $A$, The intermediate target was closer to the initial target. $B$, The intermediate target was closer to the final target. See Figure 5 legend for description.

is required, nor is it necessary to make any assumption about the form of the neural input signals, as in some other proposed models which attempt to account for movement kinematics (Zangenmeister et al., 1981). Hence, the success of the model presented here supports the theories which view the motor system as being divided between higher levels which plan ideal trajectories for the endeffector, and lower level processes which translate them into torques and forces. These theories suggest that at higher levels there exists a kinematic representation of movement which does not take into account the mechanical nature of the actual effectors (Bernstein, 1967).

The optimization model offers a new insight into these theories: the minimum-jerk movement is independent of the neuromuscular dynamics only if the demands of the movement lie within the capabilities of the neuromuscular system. If one (or more) of the neuromuscular performance limits are reached, they impose a constraint on the achievable movements, and the planning and execution processes cannot be clearly separated. However, dynamic optimization theory is sufficiently general to cover the case of performance-limited movements and may permit a prediction of the interaction between movement kinematics and neuromuscular dynamics as the limits of performance are approached, for example, during fast movements.

During the movement of a multijoint limb, the generation of appropriate joint torques for a trajectory is complicated by the presence of significant joint interactions due to inertial, centripetal, and coriolis forces (Hollerbach and Flash, 1982). Since these interaction effects are not present in the case of single-joint movements, strategies for the solution of dynamic problems for single-joint movements may not generalize to multijoint movements. By contrast, 

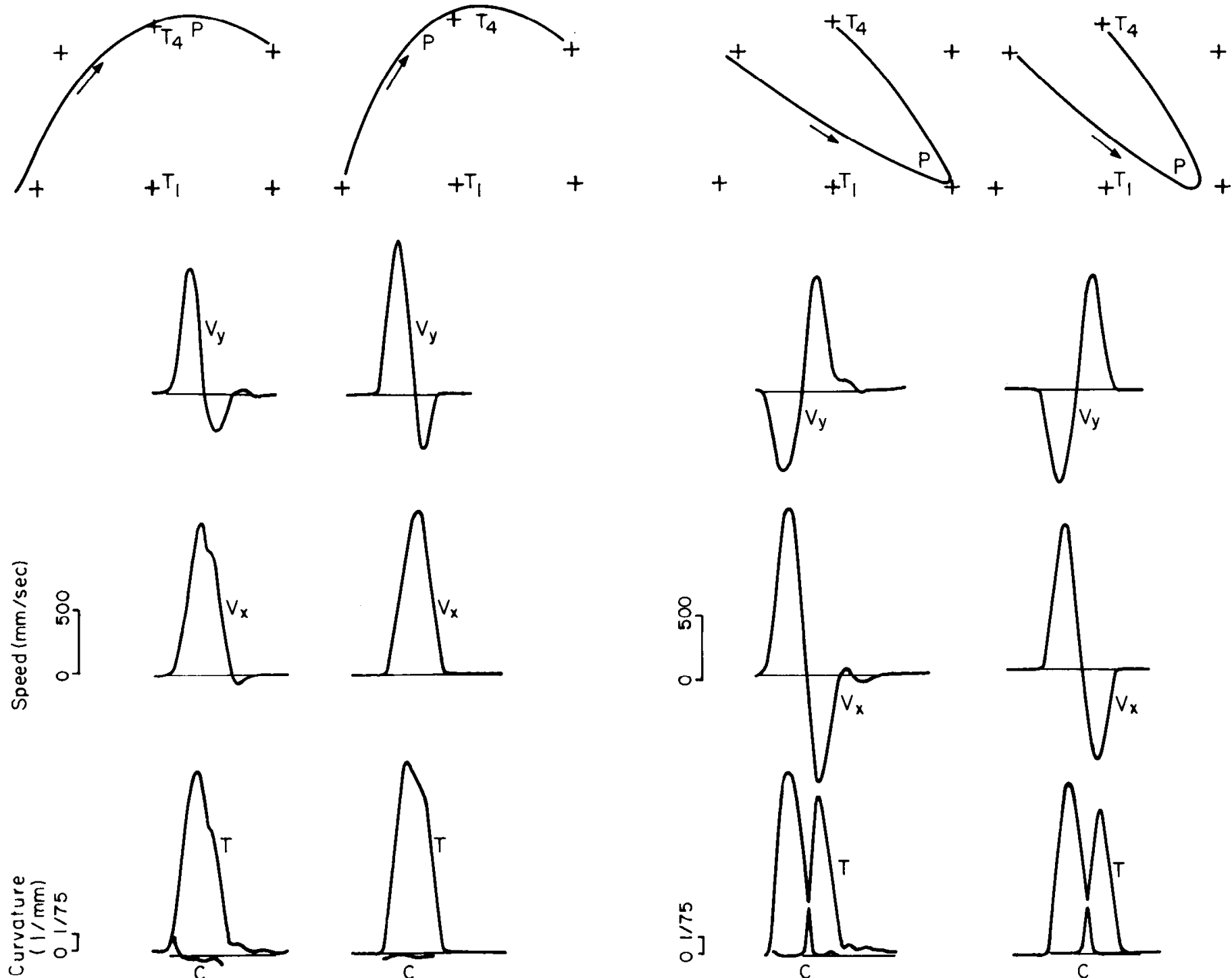

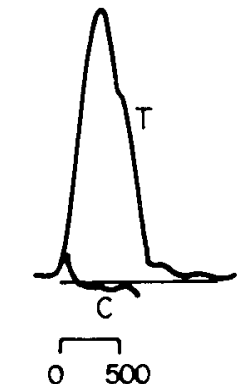

Time (msec)

Real

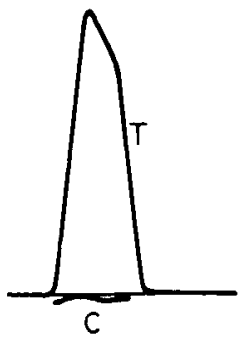

Model

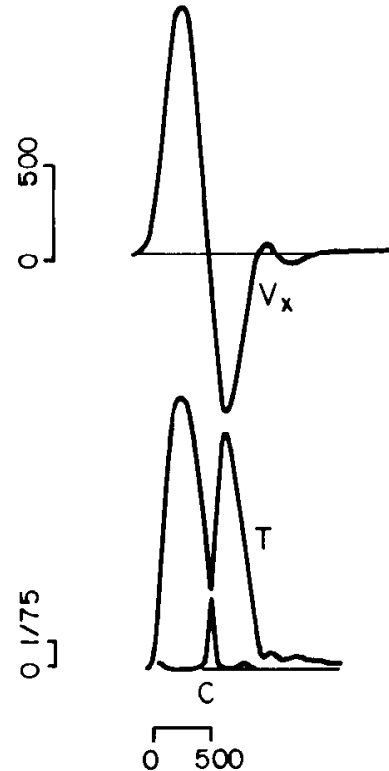

Real
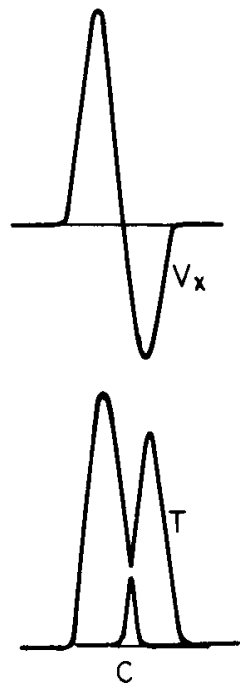

Model

Figure 7. Representative examples of comparisons between measured (Real, left columns) and predicted (Model, right columns) trajectories from a "via-point" experiment in which the movement reversed its direction along both $x$-and $y$-axes. See Figure 5 legend for description.

the minimization of mean-squared jerk has been applied successfully to both single-joint (Hogan, 1982, 1984) and multijoint movements (Flash, 1983). By an appropriate choice of the boundary conditions (i.e., the acceleration at the end of the motion is not constrained to be zero), the minimum-jerk model can also produce an excellent fit to observed repetitive movements (Nelson, 1983). Similarly, curved and straight hand movements were predicted here from the same criterion function, while al the dynamic level, each different movement requires very different patterns of joint torques. This indicates that a single unifying principle underlies the planning and the kinematic representation of all of these movements. These results also indicate that at the higher levels, a small number of general principles may be applied to the planning and coordination of movement kinematics, whereas at the lower levels, less general strategies may be used to compensate for the difference in the dynamics of multijointed and single-jointed systems.

It is important to note that, in the mathematical analysis presented here, the minimum-jerk criterion function is expressed as the sum of the squares of the third derivatives of the coordinates of the hand in the extracorporal space. If the criterion function were expressed in joint coordinates (as the sum of squares of the third timederivatives of the joint anigles), the optimization would predict trajectories which exhibit invariances in joint coordinates. Because of the complicated dependency of hand position on joint angles, such invariances are incompatible with the observed kinematic features and invariances in hand trajectory (Abend et al., 1982; Morasso, 1981). Consequently, this work provides strong support for the hypothesis that movements are planned in terms of the motion of a "disembodied" hand moving in extracorporal space.

The concept of hierarchical motor planning (Saltzman, 1979; Keele, 1981) suggests also that the same general and abstract internal representation of movement is used each time a movement is generated with temporal and spatial parameters chosen for that specific movement. Movement durations and spatial position cues were suggested as such specifiers (Keele, 1981). This would allow the CNS to use the same reference spatial coordinates for coding both visual information and motor actions and for learning, slorage, and retrieval of information in other skills such as drawing or hand- 

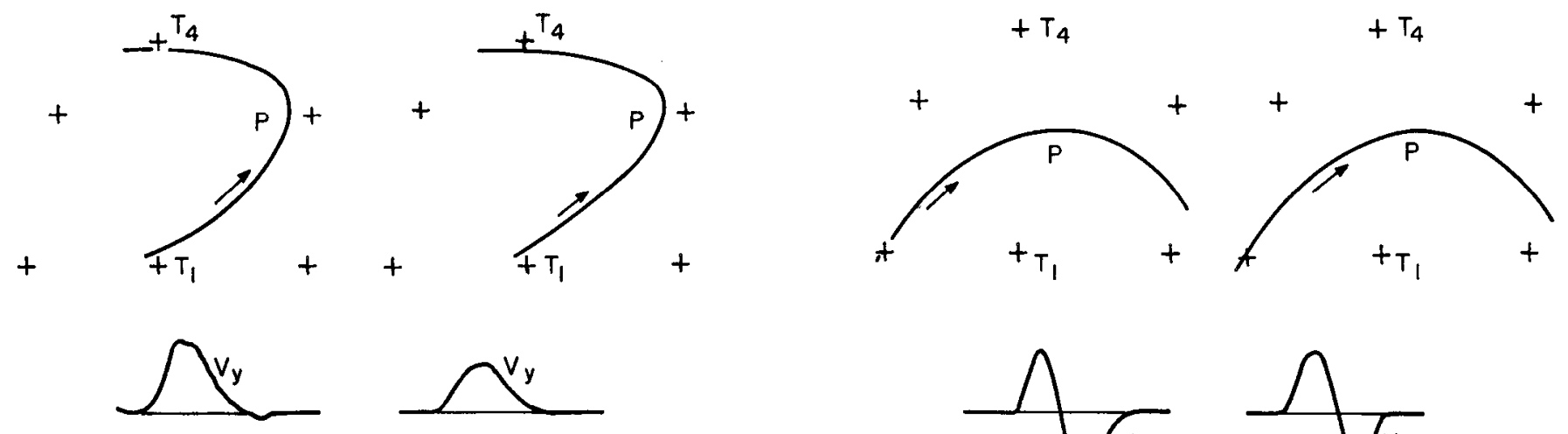

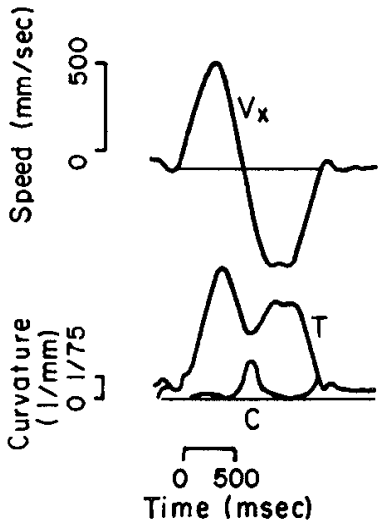

Real

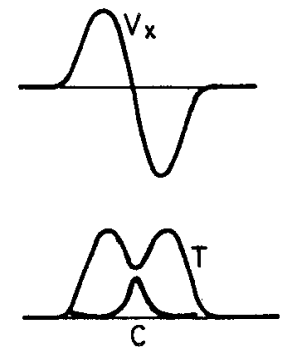

Model
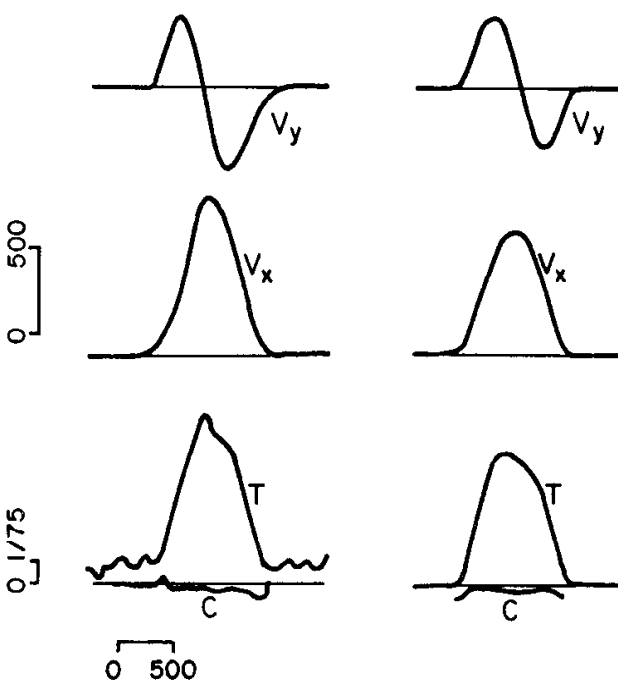

Real
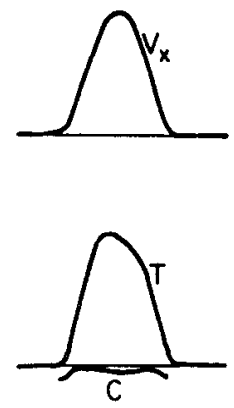

Model

Figure 8. Representative examples of comparisons between measured (Real, left columns) and predicted (Model, right columns) trajectories for two obstacle-avoidance movements.

writing. The minimum-jerk model is completely consistent with this hypothesis. The information necessary to obtain a detailed specification of the trajectory of the hand between the two targets was the movement duration and the extracorporal (e.g., Cartesian) locations of the targets.

The fact that hand trajectories are seldom performed twice in exactly the same way can be used to argue that different rules are used each time a movement is generated. Such variability in hand trajectories generated by different subjects or the same subject on succeeding trials may, however, be due to slight changes in the perceived locations of points in space to which the hand moves or through which it passes. Another possible source of movement variability might be motor variability, since the final product depends on the translation of the motor plan into muscular activity and on the interaction of the arm with the environment (Keele, 1981).

Accordingly, one physiological interpretation for the qualitative and quantitative fit of the experimentally recorded movements to minimum-jerk movements is that the CNS explicitly uses a trajectory planning strategy, which is captured by the mathematical model presented here, in order to translate task objectives, coded by exteroceptive position cues, into trajectory plans. An alternative explanation is that the smoothness of hand trajectories is an outcome of the intrinsic properties of the neural and muskuloskeletal hardware. This alternative explains the observations at the level of the neuromuscular structure rather than at the level of higher cognitive processes, but the basic principle is the same: that evolutionary adaptations have led to an optimization of biological movements. However, as mentioned above, the model presented here is based on the jerk of the hand in extracorporal coordinates and is unsuccessful when expressed in joint angles. Therefore, it can be argued that the smoothness of contraction of individual muscles cannot be the cause of the agreement between theory and experiment.
The rationale for jerk minimization in biological trajectory planning does not lend itself to self-evident, casual explanations. Given the fact that the movements under consideration occur at moderate speeds and do not subject the system to undue stress, it is unlikely that such a strategy has evolved to minimize the "wear and tear" on the system. It is possible that the objective is to minimize unwanted, abrupt changes in the forces transmitted to objects carried by the hand. Another possibility (Hogan, 1984) is that the objective is to maximize the predictability of the trajectory, which is consistent with minimizing its higher time-derivatives. To discriminate between these and other possibilities will require further work.

Finally, it is not suggested that minimization of jerk is the single objective underlying all movements. Minimization of mean-squared jerk is a mathematical model of one movement objective, the production of smooth, graceful movements. Alternatives such as the minimization of mean-squared acceleration and mean-squared snap (the fourth time-derivative of position) have been explored (Flash, 1983). This analysis has demonstrated that the minimization of meansquared acceleration results in parabolic tangential velocity profiles and non-zero accelerations at the movement end-points, unlike the actual movements. On the other hand, minimization of mean-squared snap provides a good fit to the observed experimental data. The limited resolution of the experimental data, however, has not allowed us to establish unequivocally which one of the two models, jerk minimization or snap minimization, offers a better fit. Further pursuit of the question of what objectives are optimized in human movements may help in clarifying what other principles underly motor planning (Pew and Baron, 1978).

\section{Appendices}

The optimization procedures used in this work are described in the following appendices. A comprehensive introduction to dynamic 
$+T_{4}$

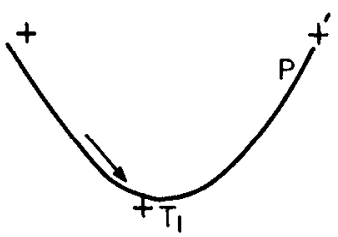

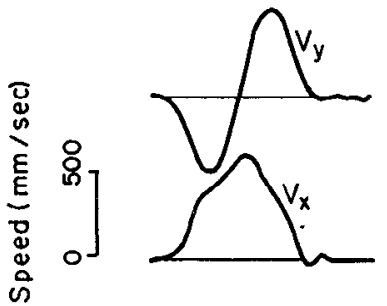
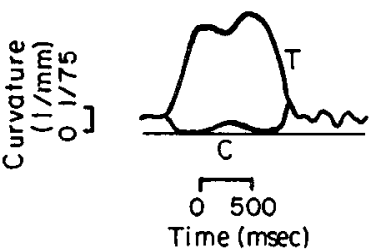

Real
$+T_{4}$
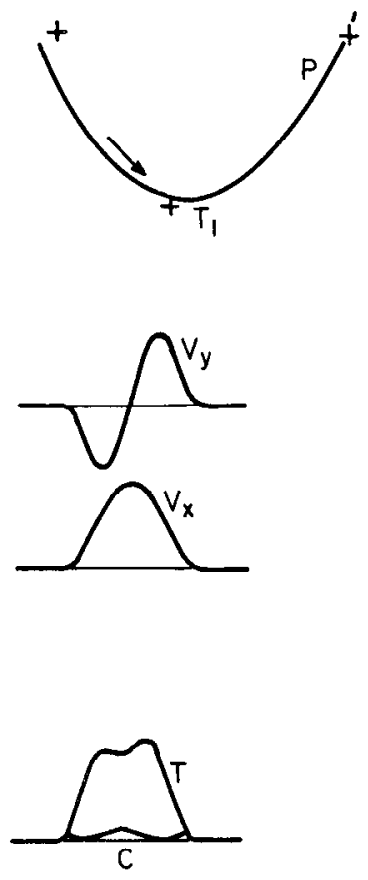

Model
$+T_{4}$

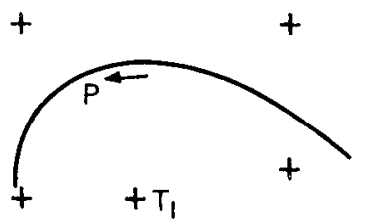

$+T_{1}$

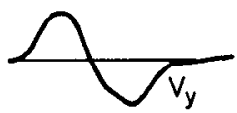

8
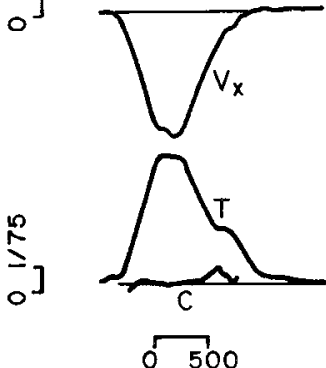

Real
$+T_{4}$
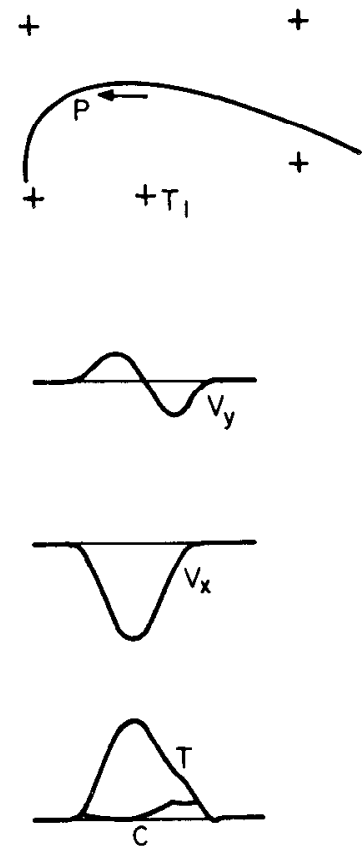

Model

Figure 9. Representative examples of comparisons between measured (Real, left columns) and predicted (Model, right columns) trajectories for two unconstrained curved movements.

optimization and variational calculus is found in Bryson and Ho (1975) and Pontryagin et al. (1962).

\section{Appendix: A: Unconstrained Optimization}

Unconstrained point to point movements. We want to minimize the following objective function:

$$
C=\frac{1}{2} \int_{0}^{t_{s}}\left(\left(\frac{d^{3} x}{d t^{3}}\right)^{2}+\left(\frac{d^{3} y}{d t^{3}}\right)^{2}\right) d t
$$

Generally, for any function $x(t)$, which is sufficiently differentiable in the interval $0 \leq t \leq t_{f}$, and for any performance index $L[t, \dot{x}, \ddot{x}, \ldots \ldots$ $\left.d^{\prime \prime} x / d t^{n}\right]$ which is integrable over the same interval, the unconstrained cost function:

$$
C(x(t))=\int_{0}^{l s} L\left[t, x, \dot{x}, \ddot{x}, \ldots \frac{d^{n} x}{d t^{n}}\right] d t
$$

assumes an extremum when $x(t)$ is the solution of Euler-Poisson equation:

$$
\frac{\partial L}{\partial x}-\frac{d}{d t}\left(\frac{\partial L}{\partial x}\right) \ldots \ldots+(-1)^{n} \frac{d^{n}}{d t^{n}} \frac{\partial L}{\partial\left(x^{n}\right)}=0
$$

Since in our case

$$
L=1 / 2\left((\ddot{x})^{2}+(\ddot{y})^{2}\right)
$$

we get the equation

$$
\frac{d^{3}}{d t^{3}}\left(\frac{\partial \ddot{x}^{2}}{\partial \ddot{x}}\right)+\frac{d^{3}}{d t^{3}}\left(\frac{\partial \dddot{y}^{2}}{\partial \ddot{y}}\right)=0
$$

We can uncouple the terms depending on the two position components to get:

$$
\frac{d^{6} x}{d t^{6}}=0 \frac{d^{6} y}{d t^{6}}=0
$$

The resulting solution to differential equations of this kind is given by a fifth order polynomial:

$$
\begin{aligned}
& x(t)=a_{0}+a_{1} t+a_{2} t^{2}+a_{3} t^{3}+a_{4} t^{4}+a_{5} t^{5} \\
& y(t)=b_{0}+b_{1} t+b_{2} t^{2}+b_{3} t^{3}+b_{4} t^{4}+b_{5} t^{5}
\end{aligned}
$$

\section{Appendix B: Dynamic Optimization}

The optimization method. Generally, optimization problems similar to the problem solved here involve a system which can be described by a set of nonlinear differential equations:

$$
\underline{\dot{s}}=\underline{f}[\underline{s}(t), \underline{u(t), t]}
$$

where $\underline{s}(t)$ is an $n$ vector function of state variables and $\underline{u}(t)$ is an $m$ vector control function. The problem is to find the control $\underline{u}(t)$ which in carrying the system from an initial state $\underline{s}(0)$ to a final state $\underline{s}\left(t_{f}\right)$, the cost function $C(t)$ is optimized. $C(t)$ is defined as:

$$
C(t)=\int_{0}^{t_{f}} L[\underline{s}(t), \underline{u}(t), t] d t
$$

where $L[\underline{s}(t), \underline{u}(t), t]$ is the performance index.

This problem can be solved by the method of Pontryagin (Pontryagin et al., 1962). One defines an $n$ component co-state (Lagrange multipliers) vector $\underline{\lambda}(t)$ and a scalar Hamiltonian:

$$
H[\underline{s}(t), \underline{u}(t), t]=L[\underline{s}(t), \underline{u}(t), t]+\lambda^{T}(t) \underline{f}[\underline{s}(t), \underline{u}(t), t]
$$

The following differential equations define the necessary conditions for a minimum to exist: 
A

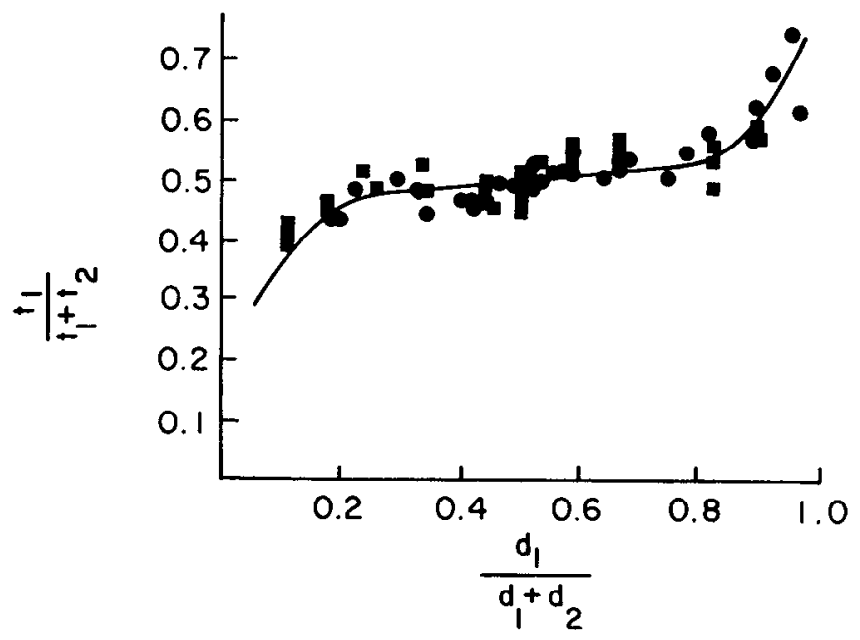

B

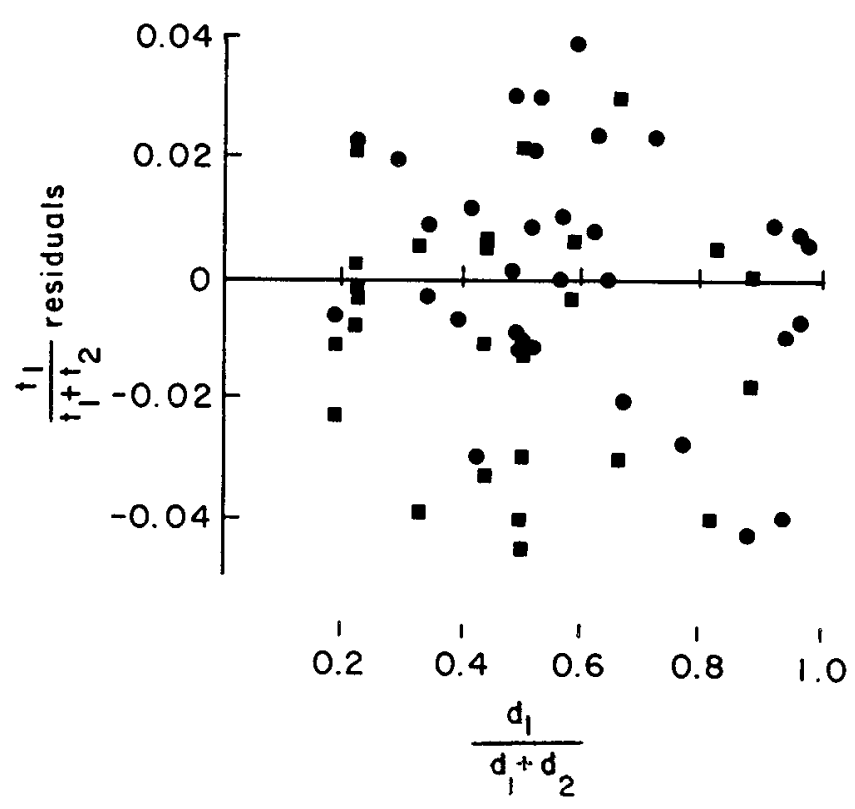

C
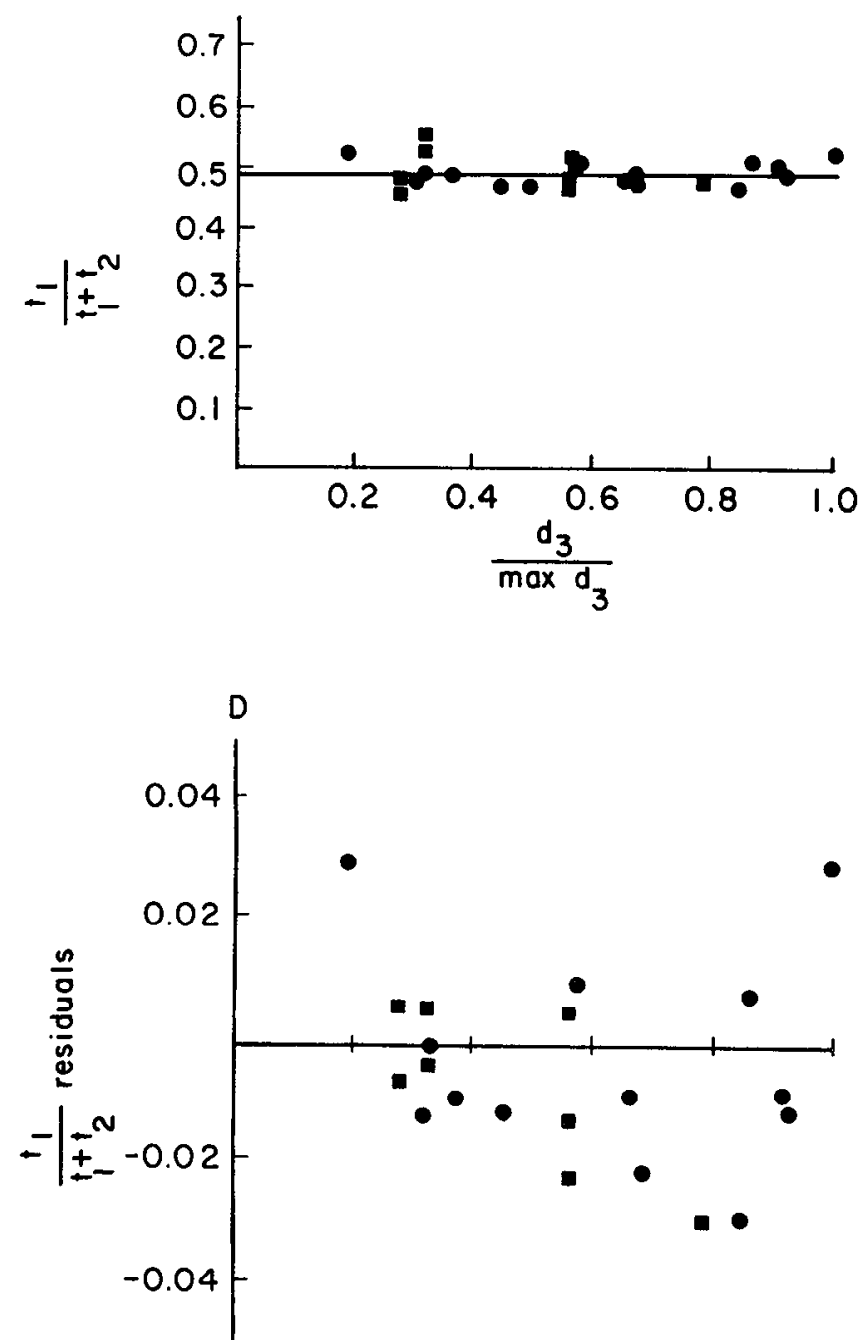

Figure 10. Parameters derived from measured unconstrained curved movements are represented by squares and parameters derived from measured movements through intermediate targets are represented by circles. $A$, A plot of the predicted $t_{1} /\left(t_{1}+t_{2}\right)$ values versus $d_{1} /\left(d_{1}+d_{2}\right)$ (solid line). Superimposed are measured $t_{1} /\left(t_{1}+t_{2}\right)$ values. $B$, A plot of the residual error between predicted and measured values of $t_{1} /\left(t_{1}+t_{2}\right)$ versus $d_{1} /\left(d_{1}+d_{2}\right)$. C, A plot of the predicted $t_{1} /\left(t_{1}+t_{2}\right)$ values versus $d_{3} /\left(\max d_{3}\right)$ (solid line). Superimposed are measured $t_{1} /\left(t_{1}+t_{2}\right)$ values. $D$, A plot of the residual error between predicted and measured values of $t_{1} /\left(t_{1}+t_{2}\right)$ versus $d_{3} /\left(\max d_{3}\right)$.

$$
\begin{gathered}
\underline{\dot{s}}(t)=\underline{f}(\underline{s}(t), \underline{u}(t), t) \\
\dot{\lambda}(t)=-\frac{\partial H}{\partial \underline{H}} \\
\frac{\partial H}{\partial \underline{u}}=0
\end{gathered}
$$

Unconstrained point-to-point movements. For our problem we define a state vector $\underline{s}^{\top}(t)=[x, y, u, v, z, w]$ and a control vector $\underline{u}^{T}(t)=[\delta, \gamma]$.

The components of these vectors are defined by the system equations:

$$
\begin{aligned}
& \dot{x}=u \\
& \dot{y}=v \\
& \dot{u}=\ddot{x}-z \\
& \dot{v}=\ddot{y}=w \\
& \dot{z}=x=j e r k_{x}=\delta \\
& \dot{w}=y=j e r k_{y}=\gamma
\end{aligned}
$$

and the Hamiltonian is:

$$
\begin{array}{r}
H=\lambda_{x} u+\lambda_{y} v+\lambda_{u} z+\lambda_{v} w \\
+\lambda_{z} \delta+\lambda_{w} \gamma+1 / 2\left(\gamma^{2}+\delta^{2}\right)
\end{array}
$$

The necessary conditions for a minimum to exist are: 


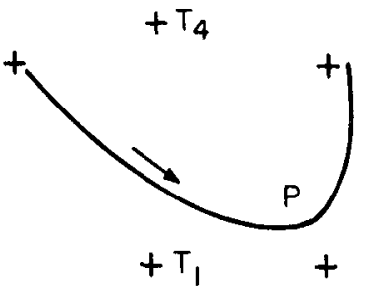

$+T^{\top}$
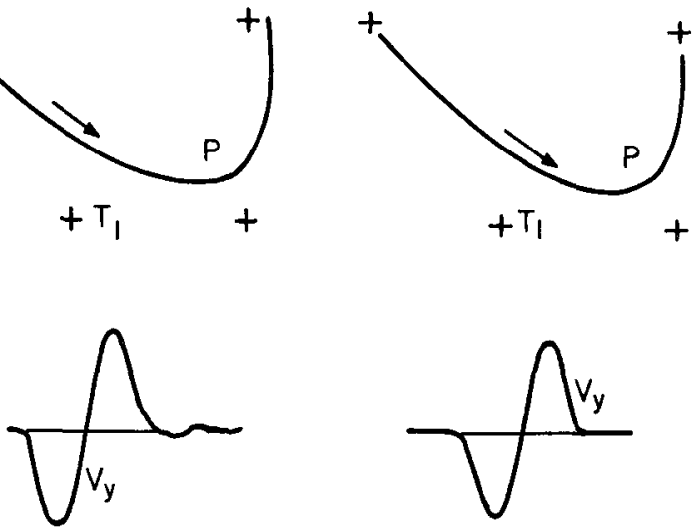

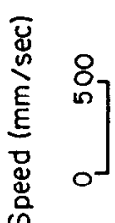

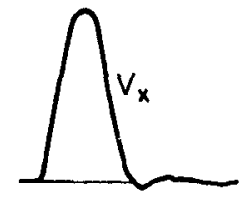

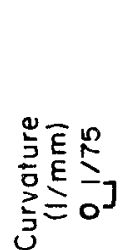

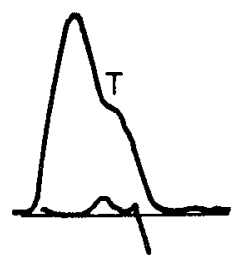

$\stackrel{500}{0}$
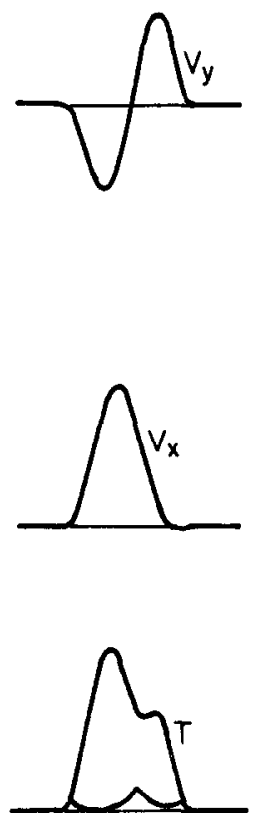

Model $+^{\top} 4$
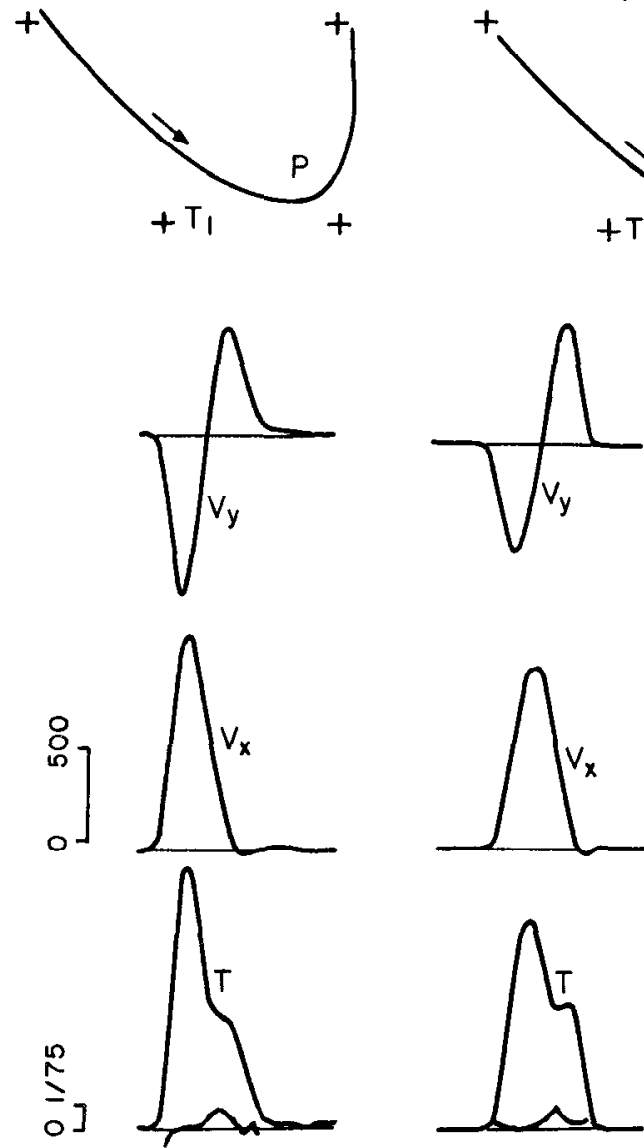

$\stackrel{5}{0} 500$

Real
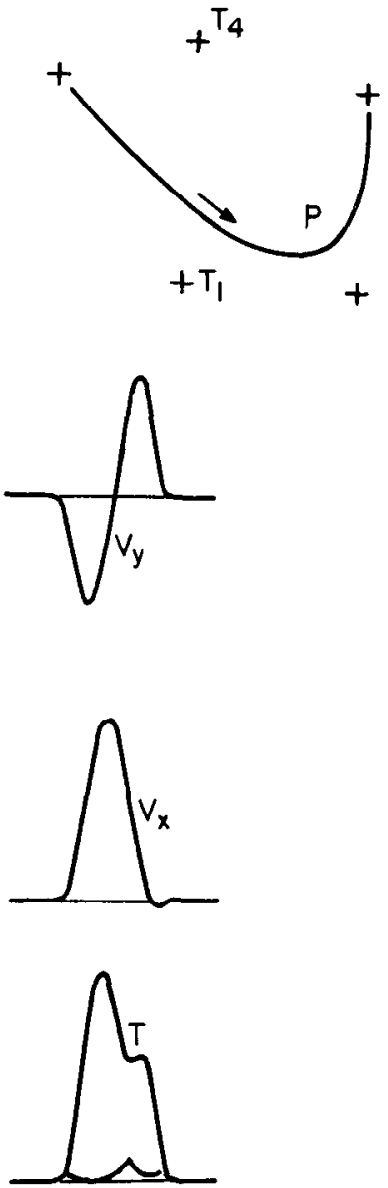

Model

A

B

Figure 11. A representative example of the scaling with speed of curved trajectories. $A, A$ 1.1-sec movement via an intermediate target. $B$, A 0.85 -sec. movement through the same targets.

$$
\begin{aligned}
-\frac{d \lambda_{x}}{d t} & =0 \\
-\frac{d \lambda_{y}}{d t} & =0 \\
-\frac{d \lambda_{u}}{d t} & =\lambda_{x} \\
-\frac{d \lambda_{v}}{d t} & =\lambda_{v} \\
-\frac{d \lambda_{z}}{d t} & =\lambda_{u} \\
-\frac{d \lambda_{w}}{d t} & =\lambda_{v}
\end{aligned}
$$

The necessary conditions on the control variables are:

$$
\begin{aligned}
& \frac{\partial H}{\partial \delta}=\delta+\lambda_{z}=0 \\
& \frac{\partial H}{\partial \gamma}=\gamma+\lambda_{w}=0
\end{aligned}
$$

Applying the following boundary conditions:

$$
\begin{array}{ll}
x(0)=x_{0} & y(0)=y_{0} \\
x\left(t_{f}\right)=x_{f} & y\left(t_{f}\right)=y_{f} \\
u(0)=0 & u\left(t_{f}\right)=0 \\
v(0)=0 & v\left(t_{f}\right)=0 \\
z(0)=0 & z\left(t_{f}\right)=0 \\
w(0)=0 & w\left(t_{f}\right)=0
\end{array}
$$


we solve these equations and get the following fifth-order polynomials for the position coordinates $x(t)$ and $y(t)$ :

$$
\begin{aligned}
& x(t)=a_{0}+a_{1} t+a_{2} t^{2}+a_{3} t^{3}+a_{4} t^{4}+a_{5} t^{5} \\
& y(t)=b_{0}+b_{1} t+b_{2} t^{2}+b_{3} t^{3}+b_{4} t^{4}+b_{5} t^{5}
\end{aligned}
$$

\section{Appendix C: Via Point Constrained Optimization}

Problems with interior point equality constaints. For curved movements we assumed that the hand is constrained to pass through the via point at time $t_{4}$ and the coordinates of this point are $x_{1}$ and $y_{1}$. Problems of this kind are defined as optimal control problems with interior point equality constraints on the state variables (Bryson and $\mathrm{Ho}, 1975$ )

For such problems one has a set of constraints at some time $t_{1}$ :

$$
\underline{N}\left(\underline{s}\left(t_{1}\right), t_{1}\right)=0
$$

where $N$ is a $q$ component vector function. These interior point constraints can be augmented to the cost function by a Lagrange multiplier vector $\pi$ so that the new cost function is:

$$
C=\underline{\pi}^{\top} \underline{N}+\int_{0}^{t_{f}}\left(H-\underline{\lambda}^{\top} \underline{\dot{s}}\right)
$$

The solution is obtained by allowing discontinuities in the co-state variables (Lagrange coefficients) $\underline{\lambda}(t)$ 's and in the Hamiltonian $H[t$, $\lambda(t), s(t)]$. One can define a vector of Lagrange coefficients $\lambda^{+}(t)$ and Hamiltonian $H^{+}(t)$ for $t \geq t_{1}$ and a vector $\underline{\lambda}^{-}(t)$ and Hamiltonian $H^{-}(t)$ for $t \leq t_{1}$. At time $t_{1}$ these variables satisfy the equations:

$$
\begin{gathered}
\underline{\lambda}^{-}\left(t_{1}\right)=\underline{\lambda}^{+}\left(t_{1}\right)+\underline{\pi}^{T} \frac{\partial \underline{N}}{\partial \underline{s}\left(t_{1}\right)} \\
H^{-}\left(t_{1}\right)=H^{+}\left(t_{1}\right)-\underline{\pi}^{T} \frac{\partial \underline{N}}{\partial t_{1}}
\end{gathered}
$$

The $q$ components of $\pi$ are determined by the constraint equations $C 1$ while time $t_{1}$ is fully determined by equation $C 4$.

Minimum-jerk trajectories with via point constraints. For our specific problem the only constraints are on hand position along both axes at time $t_{1}$ :

$$
\begin{aligned}
& x\left(t_{1}\right)=x_{1} \\
& y\left(t_{1}\right)=y_{1}
\end{aligned}
$$

The Hamiltonian $H^{\prime \prime}$ for all times, such that $t \leq t_{1}$ is:

$$
\begin{aligned}
H^{-}=\lambda_{x}^{-} u^{-}+\lambda_{y}^{-} v^{-}+ & \lambda_{u}^{-} z^{-}+\lambda_{v}^{-} w^{-} \\
& +\lambda_{z}^{-} \delta^{-}+\lambda_{w}^{-} \gamma^{-}+1 / 2\left(\left(\gamma^{-}\right)^{2}+\left(\delta^{-}\right)^{2}\right)
\end{aligned}
$$

and the Harmiltorian $H^{+}$for limes $t \geq l_{1}$ is:

$$
\begin{aligned}
H^{+}=\lambda_{x}^{+} u^{+}+\lambda_{y}^{+} v^{+}+ & \lambda_{u}^{+} z^{+}+\lambda_{v}^{+} w^{+} \\
& +\lambda_{z}^{+} \delta^{+}+\lambda_{w}^{+} \gamma^{+}+1 / 2\left(\left(\gamma^{+}\right)^{2}+\left(\delta^{+}\right)^{2}\right)
\end{aligned}
$$

Since the only constraint equations relate to position, the only discontinuities are in $\lambda_{x}$ and $\lambda_{y}$ and, therefore, according to equation C3, we get:

$$
\begin{aligned}
& \lambda_{x}^{-}\left(t_{1}\right)=\lambda_{x}^{+}\left(t_{1}\right)+\pi_{1} \\
& \lambda_{y}^{-}\left(t_{1}\right)=\lambda_{y}^{+}\left(t_{1}\right)+\pi_{2}
\end{aligned}
$$

while all the other Lagrange coefficients are continuous at $t=t_{1}$ :

$$
\begin{aligned}
& \lambda_{u}^{+}\left(t_{1}\right)=\lambda_{u}^{-}\left(t_{1}\right) \\
& \lambda_{v}^{+}\left(t_{1}\right)=\lambda_{v}^{-}\left(t_{1}\right) \\
& \lambda_{z}^{+}\left(t_{1}\right)=\lambda_{z}^{-}\left(t_{1}\right) \\
& \lambda_{w}^{+}\left(t_{1}\right)=\lambda_{w}^{-}\left(t_{1}\right)
\end{aligned}
$$

Since time $t_{1}$ is not explicitly specified, the Hamiltonian must be continuous at $t_{1}$ as emerges from equation $\mathrm{C} 4$ :

$$
H^{+}\left(t_{1}\right)=H^{-}\left(t_{1}\right)
$$

Next we write the necessary conditions for the existence of a minimum. These equations are derived separately for $t \geq t_{1}$ and $t \leq t_{1}$. In addition, we require continuity of velocities and accelerations at $t_{1}$, so that:

$$
\begin{aligned}
u^{+}\left(t_{1}\right) & =u^{-}\left(t_{1}\right) \\
v^{+}\left(t_{1}\right) & =v^{-}\left(t_{1}\right) \\
z^{+}\left(t_{1}\right) & =z^{-}\left(t_{1}\right) \\
w^{+}\left(t_{1}\right) & =w^{-}\left(t_{1}\right)
\end{aligned}
$$

These come as a result of the requirements of the optimization technique for continuity of the state variables at the interior points (Bryson and Ho, 1975).

Solving the equations, defining the necessary conditions for a minimum, and applying the boundary conditions at $t=t_{0}$ and at $t=$ $t_{f}$, one obtains an expression for $x(t)$ at all times $t \leq t_{1}$ :

$$
\begin{aligned}
& x^{-}(\tau)=\frac{t_{i}^{5}}{720}\left(\pi _ { 1 } \left(\tau_{1}^{4}\left(15 \tau^{4}-30 \tau^{3}\right)+\tau_{1}^{3}\left(80 \tau^{3}\right.\right.\right. \\
& \left.\left.\left.-30 \tau^{4}\right)-60 \tau^{3} \tau_{1}^{2}+30 \tau^{4} \tau_{1}\right)-6 \tau^{5}\right) \\
& \left.+c_{1}\left(15 \tau^{4}-10 \tau^{3}-6 \tau^{5}\right)\right)+x_{0}
\end{aligned}
$$

and for times $t \geq t_{1}$ the expression is:

$$
\begin{aligned}
x^{+}(\tau)= & \frac{t_{f}^{5}}{720}\left(\pi _ { 1 } \left(\tau_{1}^{4}\left(15 \tau^{4}-30 \tau^{3}+30 \tau-15\right)\right.\right. \\
& \left.+\tau_{1}^{3}\left(-30 \tau^{4}+80 \tau^{3}-60 \tau^{2}+10\right)\right) \\
& \left.+c_{1}\left(-6 \tau^{5}+15 \tau^{4}-10 \tau^{3}+1\right)\right)+x_{t} \\
= & x^{-}(\tau)+\pi_{1} \frac{t_{f}^{5}\left(\tau-\tau_{1}\right)^{5}}{120}
\end{aligned}
$$

where $c_{1}$ and $\pi_{1}$ are constants, $\tau=t / t_{f}$ and $\tau_{1}=t_{1} / t_{f}$.

The same expressions are obtained for $y^{+}$and for $y^{-}$and $\pi_{2}$ and $c_{2}$ replacing $\pi_{1}$ and $c_{1}$. Substituting $x^{+}\left(t_{1}\right)=x^{-}\left(t_{1}\right)=x_{1}$ in equations $C 12$ and $C 13$ and solving for $\pi_{1}$ and $c_{1}$, we obtain the following expressions:

$$
\begin{aligned}
& c_{1}=\frac{1}{t_{f}^{5} \tau_{1}^{2}\left(1-\tau_{1}\right)^{5}}\left(\left(x_{f}-x_{0}\right)\left(300 \tau_{1}^{5}-1200 \tau_{1}^{4}+1600 \tau_{1}^{3}\right)\right. \\
&+\tau_{1}^{2}\left(-720 x_{f}+\right.\left.120 x_{1}+600 x_{0}\right) \\
&\left.+\left(x_{0}-x_{1}\right)\left(300 \tau_{1}-200\right)\right)
\end{aligned}
$$

$$
\begin{aligned}
\pi_{1}=\frac{1}{t_{f}^{5} \tau_{1}^{5}\left(1-\tau_{1}\right)^{5}}\left(\left(x_{f}-x_{0}\right)\right. & \left(120 \tau_{1}^{5}\right. \\
& \left.\left.-300 \tau_{1}^{4}+200 \tau_{1}^{3}\right)-20\left(x_{1}-x_{0}\right)\right)
\end{aligned}
$$

and for $\pi_{2}$ and $c_{2}$ similarly, with $y_{0}$ replacing $x_{0}$ and $y_{i}$ replacing $x_{i}$ etc.

Next, we substitute the expressions for $\pi_{1}, \pi_{2}, c_{1}$, and $c_{2}$ in equation $\mathrm{C} 10$ which reduces to

$$
\pi_{1} u\left(t_{1}\right)+\pi_{2} v\left(t_{1}\right)=0
$$

and we get a polynomial equation in $\tau_{1}=t_{1} / t_{r}$. We find the real roots of this polynomial and accept only those roots that lie between 0 and 1 . The polynomials we obtained had only one acceptable root. We then substitute this value for $\tau_{1}$ in the expressions for $\pi_{1}, \pi_{2}, c_{1}$, and $c_{2}$ and we can firlally get the expression for $x(t)$ and $y(t)$ for the entire movement 


\section{References}

Abend, W., E. Bizzi, and P. Morasso (1982) Human arm trajectory formation. Brain 105: 331-348.

Bernstein, N. (1976) The Coordination and Regulation of Movements, Pergamon Press, Oxford.

Bizzi, E., N. Accornero, W. Chapple, and N. Hogan (1984) Posture control and trajectory formation during arm movement. J. Neurosci. 4: 27382744.

Brady, M. (1982) Trajectory planning. In Robot Motion: Planning and Control, M. Brady, J. M. Hollerbach, T. L. Johnson, T. Lozano-Perez, and M. Mason, eds., pp. 221-224, MIT Press, Cambridge, MA.

Brady, M., J. M. Hollerbach, T. L. Johnson, T. Lozano-Perez, and M. Mason (1982) Robot Motion: Planning and Control, MIT Press, Cambridge, MA.

Bryson. A. E., and Y. -C. Ho (1975) Applied Optimal Control, Hempshire Publishing Co., New York.

Clark, M. R. and L. Stark (1975) Time optimal behavior of human saccadic eye movement. IEEE Trans. on Automatic Control AC-20: 345-348.

Flash, T. (1983) Organizing principles underlying the formation of hand trajectories. Ph.D. thesis, Harvard/MIT Division of Health Sciences and Technology, Massachusetts Institute of I echnology, Cambridge, MA.

Georgopoulos, A. P., J. F. Kalaska, and J. T. Massey (1981) Spatial trajectories and reaction times of aimed movements: Effects of practice, uncertainty, and change in target location. J. Neurophysiol, 46: 725-743.

Granit, R. (1981) Comments on the history of motor control. In Handbook of Physiology. Section 1: The Nervous System, Vol. 2: Motor Control, V. B. Brooks, ed., pp. 1-16, Williams \& Wilkins, Baltimore.

Greene, P. H. (1972) Problems of organization of motor systems. Prog Theor. Biol. 2: 303-338.

Hogan, N. (1982) Control and coordination of voluntary arm movements. In Proceedings of the 1982 American Control Conference, M. J. Rabins and Y. Bar-Shalom, eds., pp. 522-528, American Automatic Control Council.

Hogan, N. (1984) An organizing principle for a class of voluntary movements J. Neurosci. 4: 2745-2754.

Hollerbach, J. M., and T. Flash (1982) Dynamic interactions between limb segments during planar arm movement. Biol. Cybern. 44: 67-77.

Keele, S. W. (1981) Behavioral anlalysis of movernent. In Hardbouk of Physiology. Section 1: The Nervous System, Vol. 2: Motor Control, V. B. Brooks, ed., pp. 1391- $14 \uparrow 4$, Williams \& Wilkins, Baltimore

Lashley, K. S. (1951) The problem of serial order in behavior. In Cerebral Mechanisms in Behavior, L. A. Jeffress, ed., pp. 112-146, John Wiley \& Sons, New York.
Morasso, P. (1981) Spatial control of arm movements. Exp. Brain Res. 42 223-227.

Morasso, P., and F. A. Mussa-lvaldi (1982) Trajectory formation and handwriting: A computational model. Biol. Cybern. 45: 131-142.

Nelson, W. (1983) Physical principles for economies of skilled movements. Biol. Cybern. 46: 135-147.

Pew, R. W. and S. Baron (1978) The component of an information processing theory of skilled performance based on optimal control perspective. In Information Processing in Motor Control and Learning, G. E. Stelmach, ed., pp. 71-78, Academic Press, Inc., New York.

Pontryagin, L. S., V. Boltyanskii, R. Gamkrelidze, and E. Mishchenko (1962) The Mathematical Theory of Optimal Processes, Interscience Publishers Inc., New York

Saltzman, E. (1979) Levels of sensorimotor representation. J. Math. Psychol. 20: $91-163$.

Schmidt, R. A. (1980) On the theoretical status of time in motor program representations. In Tutorials in Motor Behavior, G. E. Stelmach and J. Requin, eds., pp. 145-165, Elsevier-North Holland, Publishing Co., Amslerdam.

Schmidt, R. A., N. H. Zelaznick, and J. S. Frank (1978) Sources of inaccuracy in rapid movement. In Information Processing in Motor Control and Learning, G. E. Stelmach, ed., pp. 183-203, Academic Press, Inc., New York.

Soechting, J. F., and F. Lacquaniti (1981) Invariant characteristics of a pointing movement in man. J. Neurosci. 1: /10-/20.

Soechting, J. F., and F. Lacquaniti (1983) Modification of trajectory of a pointing movement in response to a change in target location. J. Neurophysiol. 49: 548-564.

Stein, R. B. (1982) What muscle variable(s) does the nervous system contro in limb movements? Behav. Brain Sci. 5: 535-578.

Taylor, R. H. (1979) Planning and execution of straight-line manipulator trajectories. IBM J. Res. Dev. 23: 424-436.

Viviani, P., and C. Terzuolo (1980) Space-time invariance in motor skills. in Tutorials in Motor Behavior, G. E. Stelmach and J. Requin, eds., pp. 525533, Elsevier-North-Holland Publishing Co., Amsterdam.

Viviani, P., and C. Terzuolo (1982) Trajectory determines movement dynamics. Neuroscience 7: 431-437.

von Hofsten, C. (1979) Development of visually directed reaching: The approach phase. J. Hum. Movement Stud. 5: 160-178.

Wing, A. M. (1980) Response timing in handwriting. In Information Processing in Motor Control and Learning, G. E. Stelmach, ed., pp. 153-172, Academic Press, Inc., New York.

Zangenmeister, W. H., S. Lehman, and L. Stark (1981) Simulation of head movement trajectories: Model and fit to main sequence. Biol. Cybern. 41 . $19-23$ 